\title{
Excessive Oral Intake of Vitamin B12 Alters Microbe-host Interactions That Stimulate Citrobacter Rodentium Growth and Virulence in Mice.
}

\author{
Andrew J. Forgie \\ University of Alberta \\ Deanna M. Pepin \\ The University of British Columbia \\ Tingting Ju \\ University of Alberta
}

Stephanie Tollenaar

University of Alberta

Consolato M. Sergi

CHEO: Children's Hospital of Eastern Ontario

Samantha Gruenheid

McGill University

Benjamin Willing ( $\nabla$ willing@ualberta.ca )

University of Alberta https://orcid.org/0000-0003-4285-4728

\section{Research Article}

Keywords: Cobalamin, Infection, Symbiosis, Microbiota, Health, Gastrointestinal

Posted Date: February 2nd, 2022

DOI: https://doi.org/10.21203/rs.3.rs-1236545/v1

License: (c) (1) This work is licensed under a Creative Commons Attribution 4.0 International License. Read Full License 


\section{Abstract}

Background: Vitamin B12 supplements typically contain doses that far exceed the recommended daily amount, and high exposures are generally considered safe. Competitive and syntrophic interactions for B12 exist between microbes on the gut. Yet, it has to be established to what extent excessive levels affect the gut microbiota and infection resistance. The objective of this study was to evaluate the effect of excess B12 on microbial ecology and pathogen virulence. Mice were fed a standard chow diet and received either water or water supplemented with B12 (cyanocobalamin; 120 mg/day) and were subsequently challenged with Citrobacter rodentium, a mouse-specific pathogen. Infection severity was determined by body weight, pathogen load, and histopathologic scoring. Host biomarkers of inflammation were assessed in the colon before and after the pathogen challenge.

Results: Cyanocobalamin supplementation led to an enhanced pathogen colonization at day $1(P<0.05)$ and day $3(P<0.01)$ post-infection. The impact of B12 on gut microbial communities, although minor, was distinct and attributed to the changes in the Lachnospiraceae populations and reduced alpha diversity. Cyanocobalamin treatment disrupted the activity of the low-abundance community members of the gut microbiota. It enhanced the amount of interleukin-12 p40 subunit protein (IL12/23p40; $P<0.001$ ) and interleukin-17a $(\mathrm{IL}-17 \mathrm{~A} ; P<0.05)$ in the colon of naïve mice. This immune phenotype was microbedependent, and the response varied based on the baseline microbiota. The cecal metatranscriptome revealed that excessive cyanocobalamin decreased the expression of glucose utilizing genes by $C$. rodentium, a metabolic attribute previously associated with pathogen virulence.

Conclusions: Cyanocobalamin supplementation enhanced the ability of $C$. rodentium to colonize the gut by impacting the microbe-host interactions that normally help to protect against pathogen colonization. This research provides insights into vitamin B12 supplementation and highlights potential consequences of over-supplementation.

\section{Background}

Vitamin B12 (cobalamin) is a cobalt-containing corrinoid molecule required for fundamental biological processes in both humans and bacteria. It is made exclusively by microorganisms and belongs to a family of organometallic cofactors called cobamides [1]. With few exceptions, such as ruminants which depend on the biosynthesis of cobalamin by resident microbes, most animals rely on its bioaccumulation in the food chain [2]. Humans must obtain cobalamin through diet because the only significant population of microbes that could produce cobalamin resides in the colon, past the absorption site in the small intestine [3]. Dietary sources of cobalamin in humans are mainly of animal origin, but supplements, fortified food products, fermented foods, and some plants and algae are available as alternatives [4].

Cobamides are essential for bacteria, playing a significant role in supporting enzyme activity in the cytosol and as coenzyme riboswitches that regulate gene expression in the nucleus [5]. Genomic studies revealed that widespread cobalamin sharing between microbes can impact microbial growth and 
metabolism through numerous cobamide-/corrinoid- dependent enzymes and transporter proteins [6-8]. Because of this, B12 and cobamide derivatives likely play a more critical role in microbe-microbe interactions that modulate microbial ecosystems than previously understood [3]. For example, B12 uptake by the gut commensal Bacteroides thetaiotaomicron was shown in vitro to limit the production of Shiga toxin-2 produced by Enterohemorrhagic Escherichia coli 0157:H7 (EHEC) [9]. This has been attributed to reducing the ability of EHEC to use ethanolamine (a breakdown product of lipid membranes and major metabolite found in the gut) by limiting the availability of cobalamin required to activate adenosylcobalamin-dependent ethanolamine ammonia-lyase. Regulation of ethanolamine metabolism influences the growth and/or virulence of several Enterobacteriaceae and Firmicute species [10]. Therefore, it follows that competition among microbes for cobalamin can impact the activity of ethanolamine-utilizing bacteria. In addition, $B$. thetaiotaomicron creates competition by using a surfaceexposed lipoprotein, which binds cobalamin with such affinity that it can remove it from intrinsic factor, a cobalamin transport protein necessary for absorption in humans and animals [11]. However, additional competitive and syntrophic interactions may exist between microbes for cobalamin and to what extent this impacts bacterial pathogenesis in the gut remains poorly understood.

Daily oral cobalamin supplements can contain doses that far exceed (as high as 10,000 $\mathrm{mg} / \mathrm{tab}$ let) the recommended daily amount of $2.4 \mu \mathrm{g} /$ day in humans. High exposures are required when normal absorption is impeded and are generally considered safe. The practice of over-supplementing cobalamin to ensure adequate absorption is supported by the fact that no upper limit has been set for B12 supplementation. We hypothesized that excessive cobalamin supplementation alters the gut microbiota's functional activity, creating a favourable environment for pathogen colonization and pathogenesis. To test this, we supplemented B12 to mice in drinking water. We challenged the animals with Citrobacter rodentium, a natural mouse-specific pathogen that mirrors the attaching and effacing pathology seen in human EHEC infections [12]. We evaluated the direct and indirect impact of excessive amounts of cobalamin on host-microbial interactions.

\section{Methods}

\section{Mice and vitamin B12 supplementation}

Animal experiments were conducted in accordance with guidelines set by the Canadian Council on Animal Care and approved by the Animal Care and Use Committee at the University of Alberta (Edmonton, $A B, C a n a d a)$. All mice were raised and maintained under specific pathogen-free or germ-free conditions. Six to seven-week-old female $\mathrm{C} 3 \mathrm{H} / \mathrm{HeO}$ J mice (Jackson Laboratories, Maine, USA) were randomly housed four or five per cage. Eight-week old female C57BI/6J mice (University of Alberta, AB, Canada) were housed three per cage using the Tecniplast Isocage-P bioexclusion system (Buguggiate, VA, Italy). Eight-week old germ-free female C57BI/6J mice were housed in an isolator (CEP Standard Safety, McHenry, Illinois, USA) with open cages in the University of Alberta Axenic Mouse Research Unit. All mice were allowed to acclimatize for one week with ad libitum access to water and standard chow containing approximately $0.08 \mathrm{mg}$ of cyanocobalamin per kilogram of diet post-autoclaving (2020SX; Envigo-teklad, 
Indiana, USA). Calculated based on the average consumption of $5 \mathrm{~g}$ of diet per mouse per day, the standard chow diet alone contributed approximately $0.4 \mathrm{mg}$ of cyanocobalamin. Mice received filtersterilized drinking water supplemented with or without B12 in the form of cyanocobalamin (V2876, Sigma-Aldrich, St. Louis, MO, USA) at $40 \mathrm{mg} / \mathrm{ml}$, approximately 100 times the amount in the diet. The recommended daily allowance of mice is approximately $0.05 \mathrm{mg}$ /day based on a diet that contains $10 \mathrm{mg}$ of B12 per kilogram and deemed adequate [13]. In addition, different forms of B12 were investigated in conventionalized $\mathrm{C} 57 \mathrm{Bl} / 6 \mathrm{~J}$ mice by supplementing cyanocobalamin and methylcobalamin (Thermo Fisher Scientific, Massachusetts, USA) at $10 \mathrm{mg} / \mathrm{ml}$ and $40 \mathrm{mg} / \mathrm{ml}$ in drinking water. In all experiments, cages were randomly assigned to treatment groups: control (CON) and B12 supplemented (cyanocobalamin at $10 \mathrm{mg} / \mathrm{ml}, \mathrm{CNCb} 10$ and $40 \mathrm{mg} / \mathrm{ml}, \mathrm{CNCbl} 40$; methylcobalamin at $10 \mathrm{mg} / \mathrm{ml}, \mathrm{MeCbl} 10$, and $40 \mathrm{mg} / \mathrm{ml}$, MeCbl40) groups accordingly. After two weeks of water treatment, survival (SURV) and early-stage pathogen colonization (EPC) experiments were performed in $\mathrm{C} 3 \mathrm{H} / \mathrm{HeOuJ}$ mice using $C$. rodentium. A $20 \%$ loss of initial body weight was selected as a humane endpoint for mice in the SURV experiment as previously described [14]. For the EPC experiment, after two weeks of B12/water treatment, two mice from each cage were euthanized (naïve_CON \& naïve_CNCbI40). The remaining mice continued on water treatment for subsequent $C$. rodentium challenge (inf_CON \& inf_CNCb|40). To confirm the pre-challenge B12-induced phenotypes observed in $\mathrm{C} 3 \mathrm{H} / \mathrm{HeOuJ}$ mice and to test the role of microbes, we additionally treated germ-free and conventional C57BI/6J mice for two weeks.

\section{Water intake and B12 dose estimation}

A pilot study (data not shown) was conducted to determine daily water consumption of mice supplemented with B12 (cyanocobalamin) in drinking water. Cyanocobalamin at $40 \mathrm{mg} / \mathrm{ml}$ or control drinking water was provided to mice ( $n=15$; five mice per cage) ad libitum and water consumption was monitored for a week. Drinking water was changed every two days and water consumption was measured. The water consumed per cage at each timepoint was considered a replicate and the average was used to compare water intake between treatments. B12 supplemented in drinking water did not impact water consumption. Daily water intake was approximately $3 \mathrm{ml}$ per mouse per day. According to water intake, B12 supplemented in drinking water at $10 \mathrm{mg} / \mathrm{ml}$ and $40 \mathrm{mg} / \mathrm{ml}$ were estimated to reach a total dose of $30 \mathrm{mg}$ and $120 \mathrm{mg}$ per mouse a day, respectively.

\section{C. rodentium-challenge model}

From a glycerol stock, C. rodentium (DBS100) was plated on MacConkey agar (BD Difco, NJ, USA) and a single colony was picked and incubated overnight at $37^{\circ} \mathrm{C}$ in Luria-Bertani broth (Sigma-Aldrich) with shaking at $200 \mathrm{rpm}$. Mice were infected with $100 \mu \mathrm{l}$ of the overnight culture $\left(1 \times 10^{9} \mathrm{CFU} / \mathrm{ml}\right)$ by oral gavage. All mice were confirmed to be free of coliforms by plating a fecal sample on MacConkey agar prior to infection. Pathogen load was determined by plating serial dilutions in 1 x PBS on MacConkey agar. Plates were incubated at $37^{\circ} \mathrm{C}$ overnight and colonies were counted and normalized to sample weight. 


\section{Sample collection}

Fresh fecal samples were collected daily or every second day post-infection directly in $1 \mathrm{ml}$ of sterile $1 \mathrm{x}$ PBS for plating. All mice were euthanized using carbon dioxide and sampling was done aseptically. Prior to infection, fecal samples were collected from mice for baseline microbiome analysis. Mouse tissues and intestinal content (ileum, cecum and colon) were snap-frozen in liquid nitrogen and stored at $-80^{\circ} \mathrm{C}$ until use.

\section{Intestinal vitamin B12 level measurement}

Snap-frozen cecal and colonic digesta samples were weighed and homogenized with two rounds of beating (30 s at $4 \mathrm{~m} / \mathrm{s}$ with a cooling step on ice) in a proprietary buffer provided by Calgary Laboratory Services: Diagnostic and Scientific Research Centre (Calgary, AB, Canada). Samples were subsequently centrifuged at $10,000 \mathrm{rpm}$ and the supernatant was collected and stored at $-20^{\circ} \mathrm{C}$. Vitamin B12 was quantified via electrochemiluminescence using the Roche Diagnostics Vitamin B12 II assay performed on the Roche Diagnostics e602 (Calgary Laboratory Services). This technique measures total vitamin B12 using a ruthenium-label recombinant porcine intrinsic factor as the reporter probe.

\section{Short-chain fatty acids analysis}

Snap-frozen cecal content was thawed on ice, weighed (30 mg/sample) and homogenized in $600 \mathrm{ml}$ of $25 \%$ phosphoric acid. Samples were centrifuged at $15,000 \mathrm{rpm}$ at $4^{\circ} \mathrm{C}$ for $10 \mathrm{~min}$ and the supernatant was passed through a $0.45 \mathrm{~mm}$ syringe filter (Fisher). A $200 \mathrm{ml}$ aliquot of filtered sample was combined with $50 \mathrm{ml}$ of internal standard (23 mmol/ml, isocaproic acid) and analyzed on a Scion 456-GC instrument.

\section{Cecal microbial metatranscriptome analysis}

Total RNA was extracted from frozen cecal samples as previously described [15]. Approximately $50 \mathrm{mg}$ of frozen cecal content was added to $0.1 \mathrm{~mm}$ glass bead-containing tubes (PowerBead Tubes, Qiagen) prefilled with $300 \mathrm{ml}$ RLT buffer (RNeasy mini kit, Qiagen) supplemented with $\beta$-mercaptoethanol (10 $\mathrm{ml} / \mathrm{ml}$, Sigma-Aldrich) and $1 \mathrm{ml}$ Trizol (Invitrogen). Cell disruption was accomplished using a FastPrep ${ }^{\hat{a}}-24$ bead-beating machine (MP biomedicals) with two rounds of beating (30 s at $6.5 \mathrm{~m} / \mathrm{s}$ ). After incubating for $5 \mathrm{~min}$ at room temperature, samples were centrifuged $\left(1 \mathrm{~min}, 12,000 \times \mathrm{g}, 4^{\circ} \mathrm{C}\right)$ and supernatants were transferred into tubes containing $300 \mathrm{ml}$ of chloroform, vortexed and incubated for 3 min. After centrifugation ( $15 \mathrm{~min}, 12,000 \times \mathrm{g}, 4^{\circ} \mathrm{C}$ ), the upper aqueous phase was carefully collected and transferred into a new tube containing $1 \mathrm{ml}$ of freshly prepared $70 \%$ ethanol solution, mixed by pipetting, and loaded onto a RNeasy spin column (RNeasy mini kit, Qiagen). RNA extraction and on-column DNA digestion (Qiagen) were completed as described by the manufacturer's protocol. The quality and quantity of RNA were measured using an Agilent Bioanalyzer. Samples with an RNA integrity number (RIN) ${ }^{3} 7.0$ were used to generate metatranscriptome libraries at Génome Québec Innovation Centre (Montréal, QC). Samples were diluted to $100 \mathrm{ng} / \mathrm{ml}$ and host rRNA-depletion (NEBNext ${ }^{\mathrm{O}}$ Human/Mouse/Rat) was 
conducted. The libraries were sequenced as 100 bp paired-end reads on a NovaSeq 6000 system (Illumina).

Analysis of unfiltered raw data ( $35 \mathrm{M}$ read average per sample) was completed using the Simple Annotation of Metatranscriptomes by Sequence Analysis 2.0 (SAMSA2) pipeline [16] as follows: PEAR (version 0.9 .10 ) to merge reads, Trimmomatic (version 0.36 ) to trim low quality reads, ShortMeRNA (version 2.1) to remove rRNA, and DIAMOND (version 2.0.2) to annotate mRNA data to the RefSeq database [17]. The merging step resulted in $\sim 25 \mathrm{M}$ merged reads per sample. Bacterial rRNA made up $\sim 9 \mathrm{M}$ reads per sample, and ribodepleted reads (mRNA transcripts) led to $\sim 2 \mathrm{M}$ annotated reads with 13M unknown reads per sample. In addition, the SEED Subsystems hierarchical database [18] was used to categorize and compare functional activities of the microbiota. Analysis of annotated reads was completed using the DESeq2 package and visualized in $\mathrm{R}$ with the ggplot2 package.

\section{Cytokine and chemokine assays}

Protein was extracted using a 2-cm piece of distal colon and homogenized in $300 \mu \mathrm{l}$ of Meso Scale Discovery lysis buffer with protease and phosphatase inhibitors as described in the assay protocol. The homogenates were centrifuged at 15,000 rpm for $10 \mathrm{~min}$, and the protein concentration in the supernatant was determined using the Pierce Bicinchoninic Acid assay Kit (Thermo Scientific). Sample homogenates from naïve mice and infected mice were loaded into wells at $150 \mu \mathrm{g}$ and $100 \mu \mathrm{g}$ of total protein, respectively. The U-Plex Biomarker Group 1 (mouse) assay platform (Meso Scale Discovery, Gaithersburg, MD, USA) was used to measure interferon gamma (INFY), interleukins (IL1 $\beta$, IL4, IL6, IL10, IL12/IL23p40, IL17A, and IL22), keratinocytes-derived chemokine (KC), tumour necrosis factor alpha (TNFa), granulocyte-macrophage colony-stimulating factor (GM-CSF), matrix metalloproteinase-9 (MMP9), chemokine protein known as regulated on activation normal T cell expressed and secreted (RANTES), interferon gamma-induced protein-10 (IP10), monocyte chemoattractant protein-1 (MCP1), and macrophage inflammatory proteins (MIP1a, MIP2, and MIP3a). Final concentrations were presented as $\mathrm{pg} / \mathrm{ml}$ in $100 \mu \mathrm{g}$ of total colon protein.

\section{Microbial community analyses}

Total DNA was extracted from ileum, cecum, and colon contents using the Qlamp Fast DNA Stool Mini Kit (Qiagen, Valencia, CA) with an additional bead-beating step using $\sim 200 \mathrm{mg}$ of garnet rock at $6.0 \mathrm{~m} / \mathrm{s}$ for $60 \mathrm{~s}$ on a FastPrep-24 5G instrument (MP Biomedicals). Amplicon libraries were constructed according to the protocol from Illumina (16S Metagenomic Sequencing Library Preparation) that amplified the V3-V4 region of the bacterial 16S rRNA gene: 341F (5'-

TCGTCGGCAGCGTCAGATGTGTATAAGAGACAGCCTACGGGNGGCWGCAG - 3') and 805R (5'GTCTCGTGGGCTCGGAGATGTGTATAAGAGACAGGACTACHVGGGTATCTAATCC-3'). Paired-end sequencing was accomplished using an Illumina MiSeq Platform $(2$ × 300 cycles; Illumina Inc., San Diego, CA). Raw sequences were processed with Quantitative Insight into Microbial Ecology 2 (QIIME) [19] pipeline using the divisive amplicon denoising algorithm 2 (DADA2) to filter, trim and merge paired-end reads into amplicon sequence variants (ASVs). Ribosomal RNA data from cecal 
metatranscriptomic sequencing was processed as pre-merged single-end reads in QIIME2 using deblur denoise-16S function and trimmed at $160 \mathrm{bp}$. Phylogenetic trees were constructed using the qiime alignment (mafft; mask) and qiime phylogeny (fasttree; midpoint-root) function. Taxonomy was assigned using the qiime feature-classifier classify-sklearn function using the SILVA v138 database trained for the specific amplicon region [20]. QIIME2 files (.qza) were imported into R using qiime2R (version 0.99.4) package and analyzed with phyloseq (version 1.34.0) package [21].

Alpha diversity (Observed, Shannon, Phylogenetic diversity (PD)) and beta diversity (weighted and unweighted UniFrac) indices were analyzed with rarefied samples (ileum at 21,497, cecum at 34,012, and colon at 7,435 reads) in $\mathrm{C} 3 \mathrm{H} / \mathrm{HeO} \mathrm{J}$ mice. In addition, we analyzed the cecal microbial community by analyzing the rRNA (rarefied at 1,247,061 reads) from the metatranscriptome sequencing data. Statistical significance for alpha diversity indices was determine with ANOVA and Tukey correction. Principal coordinate analyses ( $\mathrm{PCOA}$ ) was plotted using the phyloseq package and clustering significance was determined using the 'betadisper' function [22] for dispersion and 'pairwiseAdonis.dm' function [23] for orientation. Differential abundance analysis was done with DESeq2 using non-rarefied reads and tree_glom (or tax_glom for cecum rRNA) function. The 'log2foldchange' of only the ASVs with a $P$ value less than 0.05 were plotted with bolded ASVs signifying the significant adjusted $P$ value $<0.10,<0.05\left(^{*}\right)$, $\left.<0.01{ }^{(* *}\right)$ and $\left.<0.001{ }^{(* \star}\right)$. Plotted ASVs were shorten according to their lowest classifiable taxonomic rank and are distinguishable by their corresponding ASV number assigned from most to least abundant.

\section{In vitro culture experiments}

All in vitro culture experiments were done in an anaerobic chamber $\left(5 \% \mathrm{CO}_{2}, 5 \% \mathrm{H}_{2}\right.$, and $\left.90 \% \mathrm{~N}_{2}\right)$ and cultures were incubated at $37^{\circ} \mathrm{C}$ without shaking. B. thetaiotaomicron was isolated from $\mathrm{C} 3 \mathrm{H} / \mathrm{HeOuJ}$ mice fecal samples by serially diluting in $1 \times$ PBS with $0.1 \%$ L-cysteine and plating on pre-reduced brain heart infusion (BHI; Difco) agar plus 10\% calf blood (Cedarlane, ON, Canada) supplemented with $200 \mu \mathrm{g} / \mathrm{ml}$ of gentamicin [24]. Isolates were identified by amplifying and Sanger sequencing the $16 \mathrm{~S}$ rRNA gene, and sequences were matched using BLAST web-based tool [25]. C. rodentium $(10 \mathrm{ml}$ of overnight culture) was inoculated alone or in competition with $B$. thetaiotaomicron $(100 \mathrm{ml}$ overnight culture) in $10 \mathrm{ml}$ of pre-reduced low-glucose Dulbecco's modified Eagle's medium (Gibco life technologies, Grand Island, NY, USA) supplemented with cyanocobalamin at 0 ppm, $0.01 \mathrm{ppm}$ and $15 \mathrm{ppm}$, which was subsequently incubated for 6 hours. Overnight cultures grown from a single colony of $C$. rodentium grown in Luria-Bertani broth at $1.4 \times 10^{7} \mathrm{CFU} / \mathrm{ml}$ and $B$. thetaiotaomicron grown in BHI broth (Difco) at $5.5 \times 10^{7} \mathrm{CFU} / \mathrm{ml}$ were used as inoculums. Counts were determined by plating on MacConkey agar for $C$. rodentium and the $\mathrm{BHI}$ with calf blood agar for $B$. thetaiotaomicron. Total RNA was immediately extracted from $1 \mathrm{ml}$ of pelleted cells with $1 \mathrm{ml}$ of Trizol reagent and purified using the spin columns as described above.

\section{Reverse-transcription quantitative PCR}


Colon tissues were homogenized in $600 \mathrm{ml}$ of lysis buffer via bead beating and RNA was extracted using the GeneJET RNA Purification Kit (Thermo Scientific). Sample were treated with DNase as manufacturer's protocols. RNA samples extracted from both colon tissue and in vitro culture experiment were reverse transcribed using the qFlex cDNA Synthesis Kit (Quanta Bioscience). Primers used for quantitative PCR (Table S1) were previously validated $[24,26,27]$. The qPCR was performed using PercfeCTa SYBR Green Super-mix (Quantabio) conducted on an ABI StepOne real-time System following the cycles: $95^{\circ} \mathrm{C}$ for 3 min and 40 cycles of $95^{\circ} \mathrm{C}$ for $10 \mathrm{~s}, 60^{\circ} \mathrm{C}$ for $30 \mathrm{~s}$. Gene expression was calculated using the delta-delta $\mathrm{Ct}$ $\left(2^{-\Delta \Delta C t}\right)$ method that showed the fold change relative to a housekeeping gene.

\section{Statistical analysis}

Significance testing was conducted using GraphPad Prism 6 (Graphpad Software, La Jolla, CA, USA). Student's t-test or ANOVA was used for parametric and Kruskal-Wallis test was used for nonparametric data. Data were presented as mean \pm standard deviation. Survival curve analysis was done using MantelCox test with data up to day 10 post-infection. Differences between multiple treatments were corrected by conducting either the Bonferroni's, Tukey's, or Dunn's post-hoc comparison test.

\section{Results}

Cyanocabalamin supplementation enhances early-stage colonization of $\mathrm{C}$. rodentium and pathogenesis in $\mathrm{C} 3 \mathrm{H} / \mathrm{HeOUJ}$ mice.

The amount of total cobalamin in cecum and colon contents of mice were determined to be 1000 times greater $(P<0.01)$ in mice supplemented cyanocobalamin at $40 \mu \mathrm{g} / \mathrm{ml}$ in drinking water compared to control water (Fig. 1a). Higher cobalamin levels resulted in a more rapid and consistent colonization of $C$. rodentium as determined by daily fecal enumeration (Fig. 1b), and in both ileal and cecal contents at day 5 post-infection (Fig. 1C). Differences in pathogen load were greatest at day 3 post-infection $(P<0.05)$. The more rapid and consistent colonization of $C$. rodentium seen in the early pathogen colonization (EPC) experiment was confirmed in the SURV experiment. Mice receiving cyanocobalamin in excess had an earlier onset of mortality that started at day 3 compared to day 9 in control (Fig. 1d). Survival curves between $\mathrm{CON}$ and $\mathrm{CNCb} / 40$ were significantly different at day $10(P=0.03)$, although significance was lost by day 14 post-infection $(P=0.14)$. Consistent with the increased pathogen load, mice in the inf_CNCbl40 group terminated 5 days post infection had higher colon pathology scores compared to inf_CON $(P<0.05)$ (Fig. 1e). Cyanocobalamin water supplementation alone induced no visible tissue damage prior to infection (Fig. 1e). Overall, colonization and pathogenesis of $C$. rodentium in $\mathrm{C} 3 \mathrm{H} / \mathrm{HeOuJ}$ mice was enhanced following cyanocobalamin supplementation in drinking water.

\section{Cobalamin supplementation alters the Firmicutes and Proteobacteria populations within the gastrointestinal tract of $\mathrm{C} 3 \mathrm{H} / \mathrm{HeOuJ}$ mice.}

Cyanocobalamin supplementation at $40 \mu \mathrm{g} / \mathrm{ml}$ in drinking water caused a shift in microbial composition in the cecum and colon, but not ileum, favoring Proteobacteria species and altering the dynamics of the 
low-abundance Firmicutes (Figures $2 \& 3$ ). Species richness, indicated by observed counts, and phylogenetic distance analyses showed that B12 treatment led to lower colonic diversity prior to pathogen challenge. Principal Coordinate Analysis (PCoA) plots using weighted and unweighted UniFrac distance metrics showed distinct clustering of microbiomes in naïve and infected mice (pairwise Adonis; Table S2). Differences in unweighted UniFrac, but not weighted UniFrac, revealed that low-abundance community members were impacted in the cecum $(P<0.05)$ and colon $(P<0.01)$ in naïve_CNCbl40 mice compared with naïve_CON mice. The severity of microbial disruption induced by infection was more pronounced in the inf_CNCbI40 as compared to the inf_CON group. Both weighted $(P<0.01)$ and unweighted $(P<0.05)$ UniFrac metrics revealed a difference between naïve_CNCbl40 and inf_CNCb140 in the colon, whereas no difference was observed between infected and uninfected control groups (weighted $P=1.0$, unweighted $P=0.81$ ). In the cecum, the gut microbial communities between naïve_CON and inf_CON were different $(P<0.01)$ based on unweighted UniFrac metric, whereas naïve_CNCbl40 and inf_CNCbl40 groups were different $(P<0.01)$ by weighted UniFrac metric.

Differences in beta diversity observed in the colon and cecum were largely explained by changes in Firmicute and Proteobacteria populations in both naïve and infected mice as determined by DEseq2's differential expression analysis (Fig. 3). In advance of infection, an uncultured bacterium belonging to the Clostridia vadinBB60 group and a Lachnospiraceae were significantly lower in the cecum $(P<0.001$ and $P<0.05$, respectively) and colon ( $P<0.001$ and $P<0.05$, respectively), whereas $P$ arasutterella was higher in the colon $(P<0.10)$ of the naïve_CNCbl40 group compared with the naïve_CON group (Fig. 3a). A Blautia bacterium was the only significant microbe that increased $(P<0.01)$ in the cecum but not in the colon of naïve_CNCbl40 group. Consistent with the higher fecal $C$. rodentium counts; mice in the inf_CNCb140 group had a numerically higher number of reads corresponding to $C$. rodentium compared to the inf_CON group in the ileum and colon (Fig. 3b). In the cecum, Acetatifactor and an unclassified Lachnospiraceae species increased $(P<0.05)$ while Blautia and another unclassified Lachnospiraceae species decreased $(P<0.05)$ in infected mice supplemented with cyanocobalamin. The colon of inf_CNCbl40 mice had lower levels of Blautia $(P<0.05)$ as well as numerically lower levels of species belonging to Clostridia vadinBB60 group, Oscillospiraceae, Lachnospiraceae A2, and [Eubacterium] groups compared to inf_CON. These results indicate that cyanocobalamin supplementation encourages the growth of Proteobacteria (Parasutterella and Citrobacter) species and impacts the dynamics of the low-abundance Firmicute species in the gut.

Consistent shifts in beta diversity in response to B12 supplementation were observed in the SURV and EPC experiments prior to pathogen challenge (Fig. 4). However, there were notable differences in community composition between experiments including the absence of Akkermansia municiphila, Bacteroides thetaiotaomicron, and Parasutterella species in the SURV experiments (Fig. S1), which are relevant to the immune phenotypes discussed below. Short-chain fatty acid analysis on cecal content from the SURV experiment revealed that B12 supplementation had no impact on the SCFA concentrations in the gut (Fig. S2). Despite differences in baseline microbiomes, the species richness (observed counts) and phylogenetic diversity index were lower $(P<0.05)$ in mice supplemented with B12 in both experiments (Fig. 4C). In addition to numerically lower levels of Tuzzerella species, the Clostridia 
vadinBB60 group were consistently reduced $(P<0.01)$ by B12 supplementation (Fig. $4 \mathrm{~d}, \mathrm{e})$. Due to the absence of Parasutterella in the SURV experiment, the higher relative abundance of the genus $(P<0.10)$ was only detected in the EPC experiment.

We analyzed changes in the active cecal microbiota using ribosomal RNA sequences identified in the metatranscriptome data (Fig. 5). Unweighted UniFrac showed a clear separation between the microbiota of naïve treatment groups and inf_CNCbl40 group, whereas some mice from the inf_CON group remained similar to naïve treatment groups (Fig. 5a; Table S2). No difference was observed with the weighted UniFrac analysis (Fig. 5b). Alpha diversity metrics revealed no change in observed counts or Shannon diversity indices. However, the PD metric was numerically lower in the naïve_CNCbl40 group, which was consistent with the 16S rRNA gene amplicon datasets moving from the cecum to colon. Interestingly, the inf_CNCbl40 group became more diverse $(P<0.05)$ than the naïve_CNCbl40 group as determined by the PD metric (Fig. 5C). Overall, changes in community composition with B12 supplementation and infection, including Clostridia vadinBB60 and Acetatifactor, are consistent with 16s rRNA gene amplicon data (Fig. 5d,e). Differential expression analysis of the active microbial community in the cecum did not reveal significant changes; however, does suggest restructuring of population dynamics in relation to B12 supplementation (Fig. S3).

\section{Cyanocobalamin supplementation altered the Firmicute population dynamics in the cecum}

Transcriptome analysis revealed that cyanocobalamin treatment led to changes in overall microbial activity pre- and post-pathogen challenge (Fig. 6). The naïve_CNCbl40 group had significantly lower expression of citrate:sodium symporter $(P<0.01)$ and a noteworthy decrease in methyltetrathydrofolatecorrinoid methyltransferase (unadjusted $P<0.001$, adjusted $P=0.64$ ), a cobalamin-specific enzyme (Fig. 6a). Post-pathogen challenge revealed that the inf_CNCbl40 group had lower expression of flagellin domain protein $(P<0.01)$, $3 \mathrm{~N}$ domain protein-glycosyl hydrolase family $(P<0.05)$, flagellar biosynthesis protein $\mathrm{A}(P<0.10)$, and reverse transcriptase $(P<0.10)$, whereas enzymes glucose-1-phosphate thymidylyltransferase $(P<0.01)$ and D-alanine-poly (phosphoribitol) ligase $(P<0.10)$ were enriched (Fig. 6b). The SEED subsystems pathway analysis (level 3) showed a trend for increased expression of genes related to the carotenoids pathway $(P<0.10)$ in the naïve_CON group (Fig. 7). At the same time, mice supplemented with B12 had greater expression of genes related to the lipopolysaccharide assembly $(P<0.05)$ and catechol branch of beta-ketoadipate $(P<0.10)$ pathways. Genes related to Gram-positive competence and putrescine utilization pathways were numerically higher in the naïve_CON group. In addition, coenzyme B12 biosynthesis pathways were favored in the naïve_CNCbI40 group (Fig. 7a). The main pathways enriched in the inf_CON group were related to triacylglycerol metabolism, acetyl-CoA fermentation to butyrate, and autoinducer 2 (A1-2) transport and processing (IsrACDBFGE) pathways. The inf_CNCbl40 group displayed microbial activity that favored the UDP-N-acetylmuramate from Fructose-6-phosphate biosynthesis pathway (Fig. 7b).

The majority of these changes were attributed to Lachnospiraceae species pre-infection and $C$. rodentium post-infection. Therefore, we extracted and compared the metatrasncriptome data annotated to 
"Lachnospiraceae" and "Citrobacter" (Fig. 8). Lachnospiraceae species drastically altered their activity in response to a gut environment saturated with cyanocobalamin. In naïve mice, cyanocobalamin treatment enhanced the expression of fibronectin type III domain-containing protein $(P<0.01)$ and serine/threonine transporter SstT $(P<0.05)$, along with favoring numerous notable genes: type II toxin-antitoxin system HicB family antitoxin, flagellin domain protein, inorganic pyrophosphatase, propionyl-CoA carboxylase, and ribosome-associated inhibitor A/sigma 54 modulation protein (Fig. 8a). In addition, cobalaminbinding protein and cyclic lactone autoinducer peptide genes of Lachnospiraceae were favored in the naïve_CON group (Fig. 8a). Lachnospiraceae species were more active in the inf_CON group than in the inf_CNCbl40 group, with a notable increase in ribosome-associated inhibitor A/sigma 54 modulation protein, flagellin domain protein, propinyl-CoA carboxylase, and sensor domain-containing phosphodiesterase. Of particular interest, ATP- $\operatorname{cob}(I)$ alamin adenosyltransferase and MULTISPECIES: type II toxin-antitoxin system antitoxin, RelB/DinJ family genes were enriched in the inf_CNCbl40 group (Fig. 8b). Cyanocobalamin supplementation changed the activity of $C$. rodentium and notably enhanced expression of numerous virulence genes: $E$. coli secretion protein $A(E s p A)$ and $D(E s p D)$, translocated intimin receptor (Tir), intimin, and E. coli attaching and effacing gene B (EaeB) (Fig. 8c). Interestingly, the gene related to glucose utilization, family 31 glucosidase, was favored in the inf_CON group along with transcriptional regulator HdfR and DUF4150 domain-containing protein (Fig. 8c).

Excess cyanocobalamin does not directly impact $\mathrm{C}$. rodentium virulence expression in vitro.

Previous studies have shown altered virulence expression of Shiga toxin-producing EHEC in vitro in response to $B$. thetaiotaomicron competition for cobalamin [9, 24], therefore, we tested $C$. rodentium virulence expression when grown alone or in competition at physiologically relevant concentrations of cobalamin (Fig. 9). Because luminal cobalamin levels increased from $0.01 \mathrm{ppm}$ to approximately $15 \mathrm{ppm}$ with supplementation, we evaluated how cyanocobalamin at $0 \mathrm{ppm}, 0.01 \mathrm{ppm}$ and $15 \mathrm{ppm}$ impacted $C$. rodentium growth and virulence. The competition assay revealed that $C$. rodentium maintains steady levels regardless of cobalamin exposure. In contrast, $B$. thetaiotaomicron numbers increased $(P<0.05)$ with additional cyanocobalamin at 0.01 and $15 \mathrm{ppm}$ (Fig. 9a), indicating that there was competition for B12. When $C$. rodentium was grown alone, cyanocobalamin treatment lowered $C$. rodentium abundance from 8.0 to $7.5 \log \mathrm{CFU} / \mathrm{ml}(P<0.05)$ in a dose-dependent manner (Fig. 9b). Neither the competition assay nor when $C$. rodentium was grown alone revealed a change virulence gene in the expression of virulence factors of the locus of enterocyte effacement (LEE) operon, which included the LEE-encoded regulator (Ler), Tir, and EspA, and were not found to be different in the competition assay (Fig. 9c,d) or when $C$. rodentium was grown alone (Fig. 9d).

\section{Cobalamin supplementation alters colonic cytokine profiles of naïve and infected $\mathrm{C} 3 \mathrm{H} / \mathrm{HeO} \mathrm{J}$ mice.}

To determine host response to cyanocobalamin treatment, cytokine and chemokine biomarkers were measured in the colon tissue of naïve and infected mice of the EPC experiment (Fig. 10; Fig. S4 \& S5). Naïve mice supplemented cobalamin in excess had greater concentrations of cytokines IL-12/23p40 $(P<$ 0.001), IL-4 $(P=0.06)$, and IL-17A $(P<0.05)$ in colon tissue (Fig. 10a). The inf_CNCbl40 group had 
increased levels of IFNY $(P<0.05)$, IL-10 $(P<0.05)$, IL-17A $(P<0.01)$, and GM-CSF $(P<0.05)$ compared to inf_CON group (Fig. 10b). These results suggest that cyanocobalamin supplementation impacted immune activation in advance of infection.

\section{Colonic p40 subunit increased with cobalamin treatment depending on microbiota status.}

The IL-12/23p40 measurement could represent either the IL-12 or IL-23 cytokines. Therefore, we measured gene expression of IL12A (p35) and IL12B (p40) in the colon. IL12B was significantly enriched $(P<0.01)$ in the naïve_CNCbl40 group compared the naïve_CON group, but no difference was observed with IL12A (Fig. 11a). In agreement, IL-12p70 cytokine levels, a heterodimer of p35 and p40 subunits were below the assay's detection limit in the colon (data not shown). The increase in IL-12/23p40 levels was not observed in the SURV experiment mice (Fig. 11b), indicating the response is likely dependent on a specific component of the microbiome. As such, we tested whether a microbial community was required for the induction of IL-12/23p40. Indeed, cyanocobalamin treatment did not increase IL-12/23p40 protein levels in germ-free C57BI/6J mice (Fig. 11C), although it did increase in conventional C57BI/6J mice (Fig. 11d). We also wished to rule out the potential of cyanide as compared to other forms of B12. Conventionalized C57BI/6J mice displayed similar increases in IL-12/23p40 protein levels when they received either cyanocobalamin or methylcobalamin. Colon IL12/23p40 protein levels were greatest in the CNCbl10 $(P<0.05)$ and MeCbl10 $(P<0.05)$ groups, while CNCbl40 and MeCbl40 were numerically higher (Fig. 11c).

\section{Discussion}

We demonstrate that excessive cobalamin levels in the gut can alter the functional dynamics of the microbiota and host immune signaling, providing an environment supportive of pathogen colonization. It is well established that host metabolism, including host diet, environmental factors, and immune status, drives the ecological environment of the gastrointestinal tract [28-30]. Perturbations in activities of the gut microbiota, otherwise known as a gut dysbiosis, are typically described along with several diseases $[31,32]$. A study using antibiotic treatment to deplete butyrate-producing microbes revealed that this type of dysbiosis supports Enterobacteriaceae expansion and inflammation through suppression of the peroxisome proliferator-activated receptor gamma (PPAR- $\gamma$ ) homeostatic signaling pathway [33]. The activation of PPAR-y has been shown to prevent gut inflammation by regulating macrophage and T cell populations [34,35]. Many studies using antibiotics and diet to induce a dysbiosis have associated it to increased mucosal inflammation with a shift in pathogen and/or pathobiont activity [36-39]. Environmental factors and cooperative-metabolic strategies in the host can promote host-microbiota mutualism and promote the shift of a virulent pathogen to an avirulent passenger of the gut [40]. In the present study, the effect of high cobalamin levels on gut host-microbe interactions may help to explain the enhanced $C$. rodentium colonization, reduced survival and increased mucosal damage in cyanocobalamin-treated mice. 
Cyanocobalamin treatment had a subtle impact on overall microbiota structure but a distinct impact on Firmicute populations in cecum and colon. A study using C57BI/6J mice concluded that B12 supplementation minimally impacts microbial community structure under healthy conditions, but does after DSS-induced colitis treatment [41]. Under these conditions, the researchers saw a decrease in numerous Firmicutes species, including the Lachnospiracaea family members. Still, more interestingly, they saw an increase in Enterobacteriaceae species in mice supplemented with B12. This data supports our findings that naïve mice supplemented with cyanocobalamin consistently showed decreased alpha diversity due to the reduced abundance of Firmicutes species and an increase in Parasutterella species. Upon $C$. rodentium challenge, we found that mice supplemented with cyanocobalamin had a greater quantity of $C$. rodentium with a notable change to numerous Lachnospiraceae species. In particular, cyanocobalamin treatment consistently reduced Clostridia vadinBB60 group, Lachnospiraceae NK4A136 group, and other Lachnospiraceae species in both the SURV and EPC experiments. Similar changes to the microbiota were found to increase the ability of Salmonella to colonize the gut [42]. Researchers found that gnotobiotic mice harboring greater abundances of Clostridia vadinBB60 and Lachnospiraceae NK4A136 groups, as well as Ruminococcaceae members had enhanced colonization resistance to a Salmonella challenge [42]. In humans, high levels of Clostridia vadinBB60 has also been associated with low Escherichia-Shigella [43]. The interactions among these Firmicutes and other commensal microbes have been shown to contribute to a host's ability to resist pathogen colonization and pathogenesis in Salmonella, Clostridium difficile, EHEC, and Citrobacterinfections [44-48]. Therefore, we looked at the activity of the cecal microbiota in naïve and infected mice to better understand the impact of cyanocobalamin on the integration of $C$. rodentium into the resident gut community.

The activity of the gut microbiota, as determined through cecal metatranscriptome, was altered by cyanocobalamin treatment, indicating that cobalamin transport and utilization may be altered in some microbes. The citrate:sodium symporter, which was more expressed in naïve_CON than naïve_CNCb|40 group, is present only in a few pathogenic and commensal microbes in the gastrointestinal tract, and is required for citrate fermentation [49]. Interestingly, the increased citrate utilization by the gut commensal and opportunistic pathogen Enterococcus faecalis improved their pathogenic behavior in Galleria mellonella larvae [49]. Following cyanocobalamin supplementation, the loss in citrate:sodium symporter expression suggested an overall reduction in citrate metabolism by commensal microbes. This would leave more citrate for $C$. rodentium and/or open a niche that is typically filled by citrate-metabolizing microbes in naïve mice. The persistence of commensals in various niches of the gut likely contributes to a host's ability to resist pathogen colonization through competitive exclusion [50], and the enhanced citrate metabolism could be a good indication of microbe-microbe mutualism. In addition, the increased expression of lipopolysaccharide assembly genes implies that Gram-negative microbes were more active with cyanocobalamin supplementation. This was matched by an increase in genes related to the catechol branch of beta-ketoadipate pathway, which may be related to Pseudomonas species, as they have been shown to degrade B12 [51]. The increase in 'coenzyme B12 biosynthesis' was related to genes involved in cobalamin transport, a result of microbes recycling cobalamin from excessive supplementation. In contrast, genes related to 'Gram-positive competence' were elevated in the control group, along with 
putrescine utilization and carotenoids pathways. These pathways have been associated with colonic immunity and microbial mutualism that help to maintain gastrointestinal homoeostasis [52-55]. Cyanocobalamin supplementation led to distinct changes to overall cecal microbiota activity, including changes to various enzymes, transcription regulators, and transporters, and may indirectly explain the enhanced colonization of $C$. rodentium. In agreement, culture experiments showed no direct impact on $C$. rodentium growth or virulence at physiological relevant cobalamin levels. Interestingly, $C$. rodentium had enhanced expression of 'family 31 glucosidase' in control mice, a feature that may explain the nature of this pathogen's metabolism and increased virulence gene expression with cyanocobalamin supplementation. In fact, bacterial glucose metabolism and host niche adaptations that increase glucose levels in the intestine have been shown to control pathogen virulence [28], and attenuate virulence in $C$. rodentium [40]. Moreover, B12 may directly contribute to $C$. rodentium metabolism through a unique mechanism involving microbially derived 1,2-propanediol, which has been shown to control virulence and ability to colonize the host effectively [56]. A notable change in flagellin domain protein, related to Lachnospiraceae species, may represent a greater necessity for these organisms to be motile. This may be connected to fibronectin type III domain-containing protein and serine/threonine transporter, both of which have been shown to be important for cell binding [57,58] and anabolic reactions [59], respectively. We suspect that this is a sign of niche displacement among Lachnospiraceae species and likely other Firmicutes in response to cyanocobalamin supplementation and pathogen challenge.

The cobalamin-induced changes in microbial composition and activity described above directly contributed to increased IL12/IL23p40 subunit levels. The cytokines IL-12 and IL-23 play a central role in T cell-mediated regulation, and their use as therapeutic targets has highlighted their importance to host defense and inflammatory disease [60-62]. The activation of IL $12 \mathrm{p} 40$ has previously been attributed to hypoxia-inducible factor-1, a key regulator of mucosal inflammation by controlling Th1/Th17 response [63]. Contrary to our results, researchers provide evidence of IL12p40 being protective against $C$. rodentium. Still, they noted a decrease in IL17, which in our mice was elevated in both a naïve and infected cyanocobalamin-supplemented groups. Previous studies have shown that the microbiota influences Th17 response in the gut [64] and that this intestinal inflammation can enhance pathogen colonization [65]. In fact, a study with similar microbial changes to our control mice caused by knocking out the Class I-restricted T cell-associated molecule gene was associated with lower Th17 response and reduced Salmonella colonization [42]. Although IL17 plays a key role in protecting against $C$. rodentium infection [66], we found that the increased level of IL17 and IL12/23p40 in colon tissue prior to infection was a sign of immune dysregulations and this led to greater colonization. Post-infection we found greater levels of IFNY and IL17A when supplemented with cyanocobalamin. An immune phenotype previously characterized in the cecum of mice infected with Salmonella that is dependent on the microbiota [45], but resulted in reduced colonization instead an increase, as described in this study. Enhanced IFNy, IL17A and GM-CSF production was associated with selecting commensal E. coli and was considered as hallmarks of intestinal inflammation [67]. Germ-free and SURV-experiment mice did not exhibit enhanced IL12/IL23p40 protein levels from B12 supplementation as observed in EPC-experiment mice. This suggests that the effects from cyanocobalamin are mediated through the changes to the gut microbiota, 
however, this cannot be the only factor contributing to pathogen exclusion because SURV-experiment mice supplemented B12 in excess were also more susceptible to $C$. rodentium pathogenesis.

\section{Conclusion}

In the present study, we show that cyanocobalamin induced-dysbiosis reduced key members in the Firmicute population, which brought on low-grade intestinal inflammation that ultimately enhanced $C$. rodentium colonization and pathogenesis. Excessive cobalamin levels in the gut alters mutualistic microbe-host interactions that would normally help to prevent pathogen colonization. In general, the cyanocobalamin-induced dysbiosis likely caused members belonging to Clostridia vadinBB60 and Lachnospiraceae NK4A136 groups to be displaced. This in turn caused a dysbiosis that altered the IL12p40 signaling pathway in the colon and opened a niche for $C$. rodentium colonization. This provided a gut environment that promotes $C$. rodentium metabolism to be more virulent, as determined by the decrease in glucose enzyme activity and increases in virulent gene expression.

\section{Abbreviations}

EHEC: Enterohemorrhagic Escherichia coli

SURV: survival experiment

EPC: early-stage pathogen colonization experiment

PBS: phosphate-buffered saline

SAMSA2: simple annotation of metatranscriptomes by sequence analysis 2.0

INFY: interferon gamma

IL-: interleukins

$\mathrm{KC}$ : keratinocytes-derived chemokine

TNFa: tumour necrosis factor alpha

GM-CSF: granulocyte-macrophage colony-stimulating factor

MMP9: matrix metalloproteinase-9

RANTES: regulated on activation normal T cell expressed and secreted

IP10: interferon gamma-induced protein-10

MCP1: monocyte chemoattractant protein-1 
MIP-: macrophage inflammatory proteins

ASV: amplicon sequencing variant

BHI: brain heart infusion

\section{Declarations}

\section{Ethics approval and consent to participate}

All animal work was conducted in accordance to the guidelines set by the Canadian Council on Animal Care and approved by the Animal Care and Use Committee at the University of Alberta (Edmonton, AB, Canada).

\section{Consent for publication}

Not applicable

\section{Availability of data and material}

All data generated or analyzed during the current study are included in this published article [and supplementary information files] or available in the NCBI SRA repository, BioProject: PRJNA791318.

\section{Competing interests}

Not applicable

\section{Funding}

This project was funded by the Vitamin Research Fund provided by the University of Alberta for graduate and post-doctoral fellow research. BW is supported by the Canada Research Chairs program.

\section{Authors' contributions}

AF, DP, SG, and BW conceived the study. AF and DP conducted the EPC and SURV experiment respectively. CS managed the pathology scoring on intestinal tissues. TJ and ST helped with animal work. AF wrote the manuscript. All authors have read, edited and approved the final manuscript.

\section{Acknowledgements}

We would like to additionally thank Nicole Coursen for helping with animal and sample collection, as well as Kuni Suzuki and the Macauley Lab at the University of Alberta for their expertise and help with the MesoScale Discovery technology.

\section{References}


1. Kennedy KJ, Taga ME. Cobamides. Curr Biol. 2020;30:R55-6. doi:10.1016/j.cub.2019.11.049.

2. Watanabe F, Bito T. Vitamin B12 sources and microbial interaction. Exp Biol Med (Maywood). 2018;243:148-58. doi:10.1177/1535370217746612.

3. Degnan PH, Taga ME, Goodman AL. Vitamin B12 as a modulator of gut microbial ecology. Cell Metab. 2014;20:769-78. doi:10.1016/j.cmet.2014.10.002.

4. Watanabe F. Vitamin B12 sources and bioavailability. Exp Biol Med. 2007;232:1266-74.

5. Tucker BJ, Breaker RR. Riboswitches as versatile gene control elements. Curr Opin Struct Biol. 2005;15:342-8. doi:10.1016/j.sbi.2005.05.003.

6. Nahvi A, Barrick JE, Breaker RR. Coenzyme B12 riboswitches are widespread genetic control elements in prokaryotes. Nucleic Acids Res. 2004;32:143-50. doi:10.1093/nar/gkh167.

7. Degnan PH, Barry NA, Mok KC, Taga ME, Goodman AL. Human gut microbes use multiple transporters to distinguish vitamin B 12 analogs and compete in the gut. Cell Host Microbe. 2014;15:47-57. doi:10.1016/j.chom.2013.12.007.

8. Sokolovskaya OM, Shelton AN, Taga ME. Sharing vitamins: Cobamides unveil microbial interactions. Science. 2020;369. doi:10.1126/science.aba0165.

9. Cordonnier C, Le Bihan G, Emond-Rheault J-G, Garrivier A, Harel J, Jubelin G. Vitamin B12 Uptake by the Gut Commensal Bacteria Bacteroides thetaiotaomicron Limits the Production of Shiga Toxin by Enterohemorrhagic Escherichia coli. Toxins (Basel). 2016;8:14. doi:10.3390/toxins8010014.

10. Rowley CA, Kendall MM. To B12 or not to B12: Five questions on the role of cobalamin in hostmicrobial interactions. PLoS Pathog. 2019;15:e1007479. doi:10.1371/journal.ppat.1007479.

11. Wexler AG, Schofield WB, Degnan PH, Folta-Stogniew E, Barry NA, Goodman AL. Human gut Bacteroides capture vitamin B12 via cell surface-exposed lipoproteins. Elife. 2018;7:1-20. doi:10.7554/eLife.37138.

12. Crepin VF, Collins JW, Habibzay M, Frankel G. Citrobacter rodentium mouse model of bacterial infection. Nat Protoc. 2016;11:1851-76. doi:10.1038/nprot.2016.100.

13. Nation Research Council (US) Subcommittee on Laboratory Animal Nutrition. Nutrient requirements of laboratory animals. 4th edition. Washington (DC): National Academies Press; 1995. https://www.ncbi.nIm.nih.gov/books/NBK231918/.

14. Willing BP, Vacharaksa A, Croxen $M$, Thanachayanont T, Finlay BB. Altering host resistance to infections through microbial transplantation. PLoS One. 2011;6:e26988. doi:10.1371/journal.pone.0026988.

15. Just S, Mondot S, Ecker J, Wegner K, Rath E, Gau L, et al. The gut microbiota drives the impact of bile acids and fat source in diet on mouse metabolism. Microbiome. 2018;6:134. doi:10.1186/s40168018-0510-8.

16. Westreich ST, Treiber ML, Mills DA, Korf I, Lemay DG. SAMSA2: A standalone metatranscriptome analysis pipeline. BMC Bioinformatics. 2018;19:1-11. 
17. O'Leary NA, Wright MW, Brister JR, Ciufo S, Haddad D, McVeigh R, et al. Reference sequence (RefSeq) database at NCBI: current status, taxonomic expansion, and functional annotation. Nucleic Acids Res. 2016;44:D733-45. doi:10.1093/nar/gkv1189.

18. Bokulich NA, Kaehler BD, Rideout JR, Dillon M, Bolyen E, Knight R, et al. Optimizing taxonomic classification of marker-gene amplicon sequences with QIIME 2's q2-feature-classifier plugin. Microbiome. 2018;6:90. doi:10.1186/s40168-018-0470-z.

19. Bolyen E, Rideout JR, Dillon MR, Bokulich NA, Abnet CC, Al-Ghalith GA, et al. Reproducible, interactive, scalable and extensible microbiome data science using QIIME 2. Nat Biotechnol. 2019;37:852-7. doi:10.1038/s41587-019-0209-9.

20. Bokulich NA, Kaehler BD, Rideout JR, Dillon M, Bolyen E, Knight R, et al. Optimizing taxonomic classification of marker-gene amplicon sequences with QIIME 2's q2-feature-classifier plugin. Microbiome. 2018;6:90. doi:10.1186/s40168-018-0470-z.

21. McMurdie PJ, Holmes S. phyloseq: an R package for reproducible interactive analysis and graphics of microbiome census data. PLoS One. 2013;8:e61217. doi:10.1371/journal.pone.0061217.

22. Anderson MJ. Distance-based tests for homogeneity of multivariate dispersions. Biometrics. 2006;62:245-53. doi:10.1111/j.1541-0420.2005.00440.x.

23. Martinez Arbizu P. pairwiseAdonis: Pairwise multilevel comparison using adonis. 2017.

24. Curtis MM, Hu Z, Klimko C, Narayanan S, Deberardinis R, Sperandio V. The gut commensal bacteroides thetaiotaomicron exacerbates enteric infection through modification of the metabolic landscape. Cell Host Microbe. 2014;16:759-69. doi:10.1016/j.chom.2014.11.005.

25. Boratyn GM, Camacho C, Cooper PS, Coulouris G, Fong A, Ma N, et al. BLAST: a more efficient report with usability improvements. Nucleic Acids Res. 2013;41 Web Server issue:29-33.

26. Oshikiri Y, Nara H, Takeda Y, Araki A, Nemoto N, Gazi MY, et al. Interleukin-12p40 variant form reduces Interleukin-12p80 secretion. Cytokine. 2019;120 December 2018:251-7. doi:10.1016/j.cyto.2019.05.017.

27. Kumar A, Sperandio V. Indole Signaling at the Host-Microbiota-Pathogen Interface. MBio. 2019;10. doi:10.1128/mBio.01031-19.

28. Anhê FF, Barra NG, Schertzer JD. Glucose alters the symbiotic relationships between gut microbiota and host physiology. Am J Physiol Endocrinol Metab. 2020;318:E111-6. doi:10.1152/ajpendo.00485.2019.

29. Forgie AJ, Fouhse JM, Willing BP. Diet-Microbe-Host Interactions That Affect Gut Mucosal Integrity and Infection Resistance. Front Immunol. 2019;10 August:1802. doi:10.3389/fimmu.2019.01802.

30. Cabral DJ, Penumutchu S, Reinhart EM, Zhang C, Korry BJ, Wurster JI, et al. Microbial Metabolism Modulates Antibiotic Susceptibility within the Murine Gut Microbiome. Cell Metab. 2019;30:800823.e7.

31. Kho ZY, Lal SK. The Human Gut Microbiome - A Potential Controller of Wellness and Disease. Front Microbiol. 2018;9 AUG:1835. doi:10.3389/fmicb.2018.01835. 
32. Geuking MB. The Human Microbiota and Chronic Disease. Hoboken, NJ, USA: John Wiley \&;\#38; Sons, Inc.; 2016. doi:10.1002/9781118982907.

33. Byndloss MX, Olsan EE, Rivera-Chávez F, Tiffany CR, Cevallos SA, Lokken KL, et al. Microbiotaactivated PPAR-y signaling inhibits dysbiotic Enterobacteriaceae expansion. Science. 2017;357:5705. doi:10.1126/science.aam9949.

34. Guri AJ, Mohapatra SK, Horne WT, Hontecillas R, Bassaganya-Riera J. The Role of T cell PPAR y in mice with experimental inflammatory bowel disease. BMC Gastroenterol. 2010;10.

35. UI Hasan A, Rahman A, Kobori H. Interactions between host PPARs and gut microbiota in health and disease. Int J Mol Sci. 2019;20.

36. Seregin SS, Golovchenko N, Schaf B, Chen J, Pudlo NA, Mitchell J, et al. NLRP6 Protects II10-/- Mice from Colitis by Limiting Colonization of Akkermansia muciniphila. Cell Rep. 2017;19:733-45. doi:10.1016/j.celrep.2017.03.080.

37. Buret AG, Motta J-P, Allain T, Ferraz J, Wallace JL. Pathobiont release from dysbiotic gut microbiota biofilms in intestinal inflammatory diseases: a role for iron? J Biomed Sci. 2019;26:1. doi:10.1186/s12929-018-0495-4.

38. Devkota S, Wang Y, Musch M. 43 Dietary Fat-Induced Taurocholic Acid Production Promotes Pathobiont and Colitis in IL-10-/-Mice. .... 2012;487:104-8. doi:10.1038/nature11225.Dietary.

39. Willing BP, Russell SL, Finlay BB. Shifting the balance: Antibiotic effects on host-microbiota mutualism. Nat Rev Microbiol. 2011;9:233-43. doi:10.1038/nrmicro2536.

40. Sanchez KK, Chen GY, Schieber AMP, Redford SE, Shokhirev MN, Leblanc M, et al. Cooperative Metabolic Adaptations in the Host Can Favor Asymptomatic Infection and Select for Attenuated Virulence in an Enteric Pathogen. Cell. 2018;175:146-158.e15. doi:10.1016/j.cell.2018.07.016.

41. Lurz E, Horne RG, Määttänen P, Wu RY, Botts SR, Li B, et al. Vitamin B12 Deficiency Alters the Gut Microbiota in a Murine Model of Colitis. Front Nutr. 2020;7 June:1-12. doi:10.3389/fnut.2020.00083.

42. Perez-Lopez A, Nuccio S-P, Ushach I, Edwards RA, Pahu R, Silva S, et al. CRTAM Shapes the Gut Microbiota and Enhances the Severity of Infection. J Immunol. 2019;203:532-43. doi:10.4049/jimmunol.1800890.

43. Lappan R, Classon C, Kumar S, Singh OP, De Almeida R V., Chakravarty J, et al. Meta-taxonomic analysis of prokaryotic and eukaryotic gut flora in stool samples from visceral leishmaniasis cases and endemic controls in Bihar State India. PLoS Negl Trop Dis. 2019;13:1-28. doi:10.1371/journal.pntd.0007444.

44. Jacobson A, Lam L, Rajendram M, Tamburini F, Honeycutt J, Pham T, et al. A Gut CommensalProduced Metabolite Mediates Colonization Resistance to Salmonella Infection. Cell Host Microbe. 2018;24:296-307.e7. doi:10.1016/j.chom.2018.07.002.

45. Thiemann S, Smit N, Roy U, Lesker TR, Gálvez EJC, Helmecke J, et al. Enhancement of IFNY Production by Distinct Commensals Ameliorates Salmonella-Induced Disease. Cell Host Microbe. 2017;21:682-694.e5. doi:10.1016/j.chom.2017.05.005. 
46. Ross CL, Spinler JK, Savidge TC. Structural and functional changes within the gut microbiota and susceptibility to Clostridium difficile infection. Anaerobe. 2016;41:37-43. doi:10.1016/j.anaerobe.2016.05.006.

47. Cameron EA, Curtis MM, Kumar A, Dunny GM, Sperandio V. Microbiota and Pathogen Proteases Modulate Type III Secretion Activity in Enterohemorrhagic Escherichia coli. MBio. 2018;9:1-10. doi:10.1128/mBio.02204-18.

48. Mullineaux-Sanders C, Collins JW, Ruano-Gallego D, Levy M, Pevsner-Fischer M, Glegola-Madejska IT, et al. Citrobacter rodentium Relies on Commensals for Colonization of the Colonic Mucosa. Cell Rep. 2017;21:3381-9. doi:10.1016/j.celrep.2017.11.086.

49. Martino GP, Perez CE, Magni C, Blancato VS. Implications of the expression of Enterococcus faecalis citrate fermentation genes during infection. PLoS One. 2018;13:1-18.

50. Ghoul M, Mitri S. The Ecology and Evolution of Microbial Competition. Trends Microbiol. 2016;24:833-45. doi:10.1016/j.tim.2016.06.011.

51. Scott WM, Burgus RC, Hufham JB, Pfiffner JJ. Microbial degradation of corrinoids. J Bacteriol. 1964;88:581-5. doi:10.1128/jb.88.3.581-585.1964.

52. Pointon JA, Smith WD, Saalbach G, Crow A, Kehoe MA, Banfield MJ. A highly unusual thioester bond in a pilus adhesin is required for efficient host cell interaction. J Biol Chem. 2010;285:33858-66. doi:10.1074/jbc.M110.149385.

53. Nakamura A, Kurihara S, Takahashi D, Ohashi W, Nakamura Y, Kimura S, et al. Symbiotic polyamine metabolism regulates epithelial proliferation and macrophage differentiation in the colon. Nat Commun. 2021;12:1-14. doi:10.1038/s41467-021-22212-1.

54. Tofalo R, Cocchi S, Suzzi G. Polyamines and Gut Microbiota. Front Nutr. 2019;6 February:16. doi:10.3389/fnut.2019.00016.

55. Nakamura A, Ooga T, Matsumoto M. Intestinal luminal putrescine is produced by collective biosynthetic pathways of the commensal microbiome. Gut Microbes. 2019;10:159-71. doi:10.1080/19490976.2018.1494466.

56. Connolly JPR, Slater SL, O'Boyle N, Goldstone RJ, Crepin VF, Gallego DR, et al. Host-associated niche metabolism controls enteric infection through fine-tuning the regulation of type 3 secretion. Nat Commun. 2018;9. doi:10.1038/s41467-018-06701-4.

57. Pointon JA, Smith WD, Saalbach G, Crow A, Kehoe MA, Banfield MJ. A highly unusual thioester bond in a pilus adhesin is required for efficient host cell interaction. J Biol Chem. 2010;285:33858-66.

58. Alahuhta M, Xu Q, Brunecky R, Adney WS, Ding S-Y, Himmel ME, et al. Structure of a fibronectin type III-like module from Clostridium thermocellum. Acta Crystallogr Sect F Struct Biol Cryst Commun. 2010;66 Pt 8:878-80. doi:10.1107/S1744309110022529.

59. Klewing A, Koo BM, Krüger L, Poehlein A, Reuß D, Daniel R, et al. Resistance to serine in Bacillus subtilis: identification of the serine transporter $\mathrm{YbeC}$ and of a metabolic network that links serine and threonine metabolism. Environ Microbiol. 2020;22:3937-49. 
60. Teng MWL, Bowman EP, McElwee JJ, Smyth MJ, Casanova J-L, Cooper AM, et al. IL-12 and IL-23 cytokines: from discovery to targeted therapies for immune-mediated inflammatory diseases. Nat Med. 2015;21:719-29. doi:10.1038/nm.3895.

61. Hamza T, Barnett JB, Li B. Interleukin 12 a key immunoregulatory cytokine in infection applications. Int J Mol Sci. 2010;11:789-806. doi:10.3390/ijms11030789.

62. Shi Z, Wu X, Santos Rocha C, Rolston M, Garcia-Melchor E, Huynh M, et al. Short-Term Western Diet Intake Promotes IL-23-Mediated Skin and Joint Inflammation Accompanied by Changes to the Gut Microbiota in Mice. J Invest Dermatol. 2021;:1-12. doi:10.1016/j.jid.2020.11.032.

63. Marks E, Naudin C, Nolan G, Goggins BJ, Burns G, Mateer SW, et al. Regulation of IL-12p40 by HIF controls Th1/Th17 responses to prevent mucosal inflammation. Mucosal Immunol. 2017;10:122436. doi:10.1038/mi.2016.135.

64. Ivanov II, Atarashi K, Manel N, Brodie EL, Shima T, Karaoz U, et al. Induction of intestinal Th17 cells by segmented filamentous bacteria. Cell. 2009;139:485-98. doi:10.1016/j.cell.2009.09.033.

65. Raffatellu M, Santos RL, Verhoeven DE, George MD, Wilson RP, Winter SE, et al. Simian immunodeficiency virus-induced mucosal interleukin-17 deficiency promotes Salmonella dissemination from the gut. Nat Med. 2008;14:421-8. doi:10.1038/nm1743.

66. Ishigame H, Kakuta S, Nagai T, Kadoki M, Nambu A, Komiyama Y, et al. Differential Roles of Interleukin-17A and -17F in Host Defense against Mucoepithelial Bacterial Infection and Allergic Responses. Immunity. 2009;30:108-19. doi:10.1016/j.immuni.2008.11.009.

67. Kittana H, Gomes-Neto JC, Heck K, Geis AL, Segura Muñoz RR, Cody LA, et al. Commensal Escherichia coli Strains Can Promote Intestinal Inflammation via Differential Interleukin-6 Production. Front Immunol. 2018;9 OCT:2318. doi:10.3389/fimmu.2018.02318.

\section{Figures}


a)

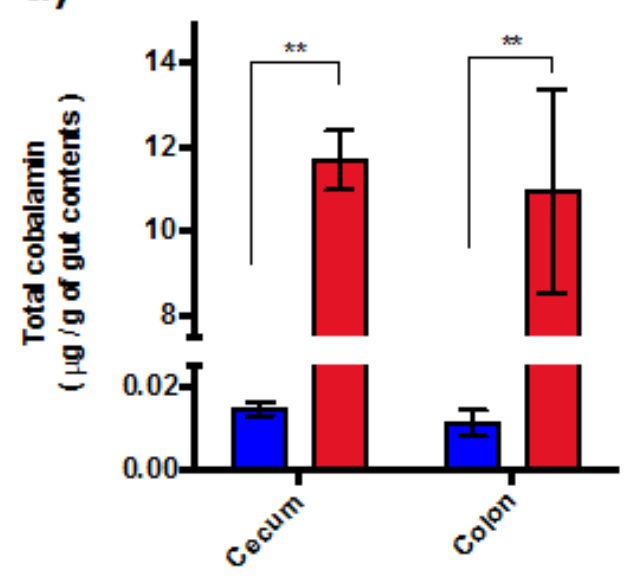

c)

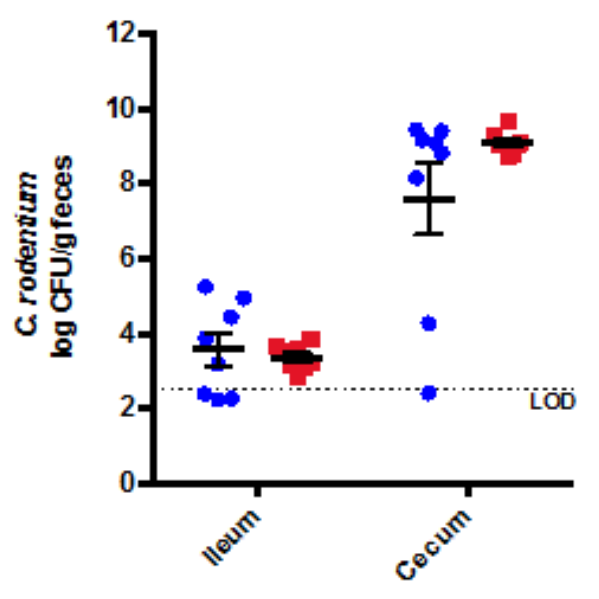

b)

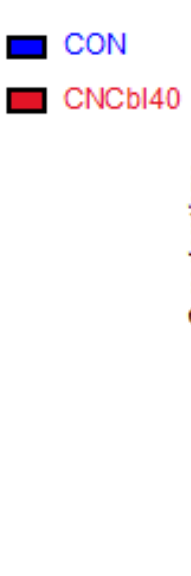

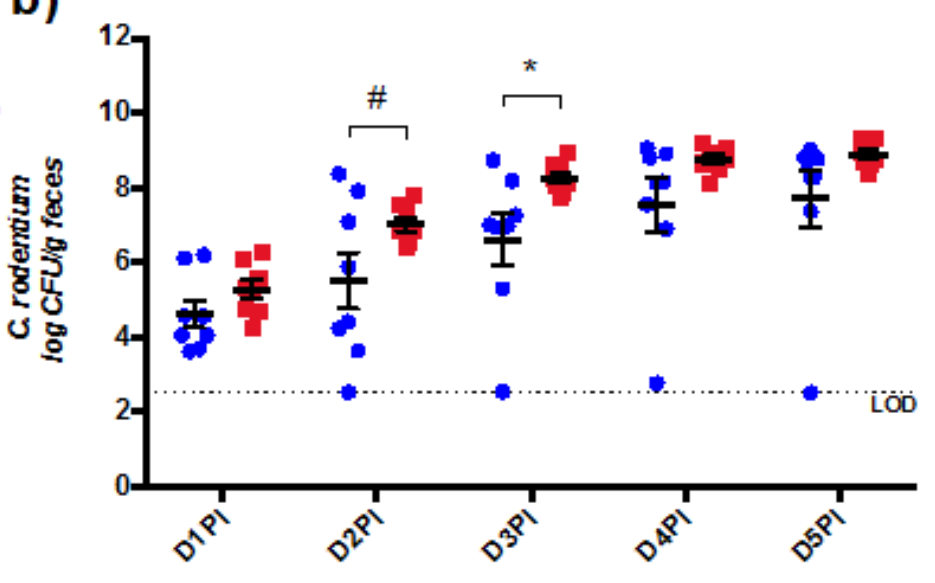

d)

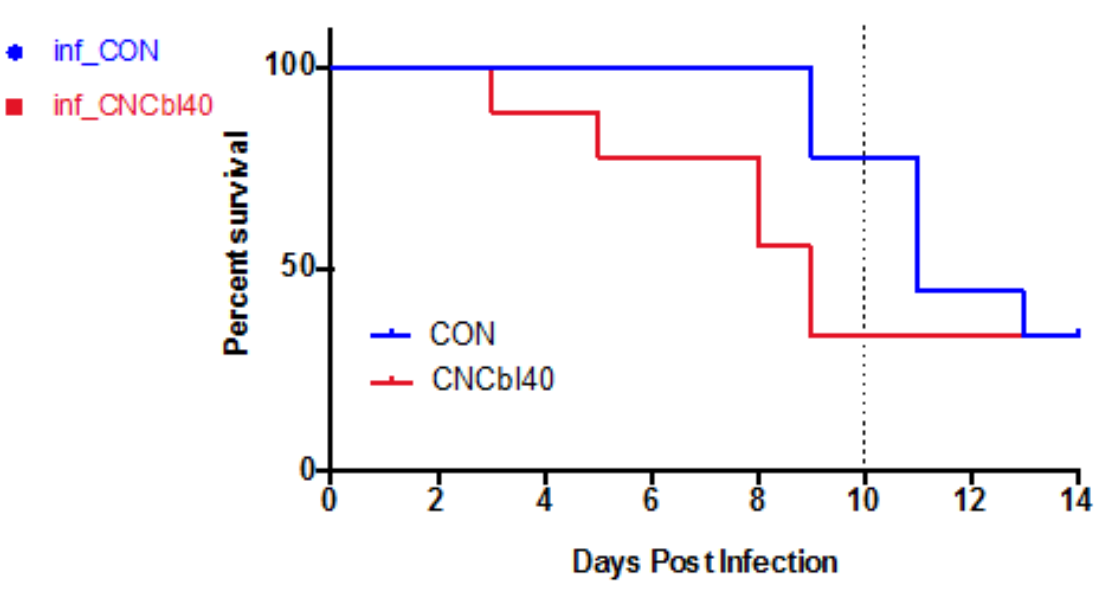

e)

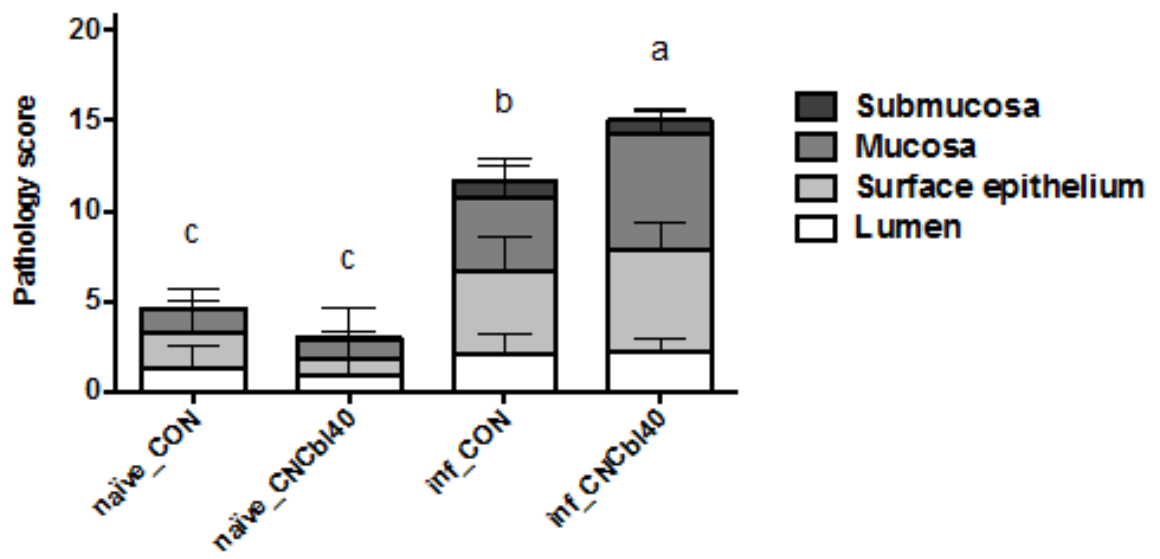

\section{Figure 1}

Cyanocobalamin supplementation enhanced $C$. rodentium colonization and pathogenesis in $\mathrm{C} 3 \mathrm{H} / \mathrm{HeOuJ}$ mice. (a) Supplementing cyanocobalamin in drinking water increased cecal and colon levels of cobalamin by $\sim 1000$ times $(\mathrm{n}=15 ; * P<0.05$, $* * P<0.01)$. (b) Daily fecal enumeration of $C$. rodentium in the EPC experiment indicated increased colonization burden at day 2 post-infection (D2PI) and D3PI from cyanocobalamin supplementation $\left(\mathrm{n}=8\right.$; $\left.\# P<0.10,{ }^{*} P<0.05\right)$. (c) Enumeration of $C$. rodentium at D5PI 
in the ileum and cecum was more consistent with cyanocobalamin supplementation but colonization levels were similar $(n=8)$. (d) Consistent with the more rapid colonization, the SURV experiment revealed an earlier onset of mortality, reducing mice survival over the first 10 days post-infection $(n=9 ; P<0.05$; Mantel-Cox test). (e) Colon pathology scores at D5PI in naïve and infected mice was significantly higher in mice supplemented with cyanocobalamin as a result of increased mucosal damage $(n=8 ; P<0.05$; limit of detection (LOD)).
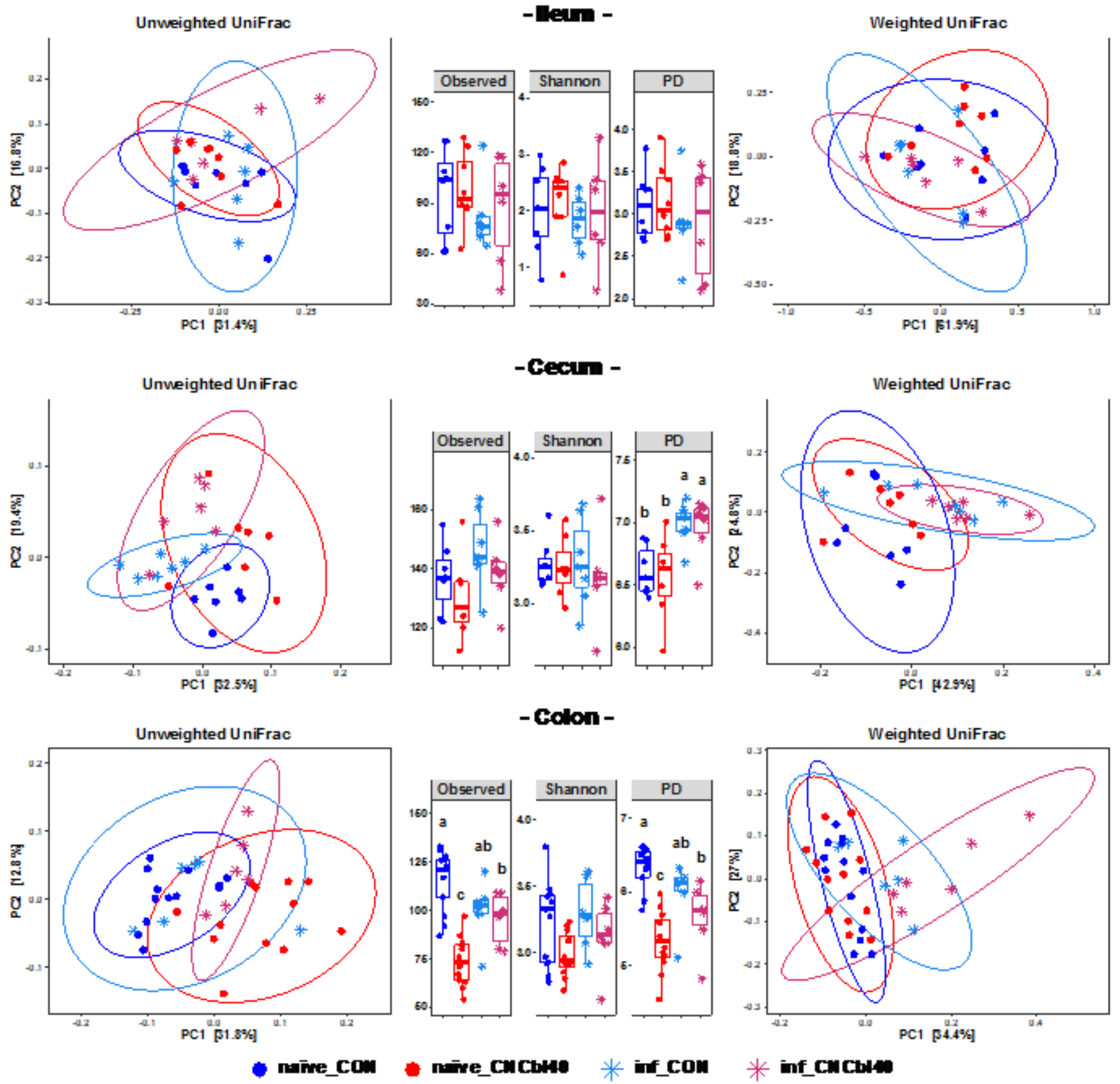

* inf_cucbur

\section{Figure 2}

Principal coordinate plots based on weighted and unweighted UniFrac and alpha diversity indices (Observed, Shannon, PD) in the ileum, cecum and colon in $\mathrm{C} 3 \mathrm{H} / \mathrm{H} 3 \mathrm{OuJ}$ mice. Distinct clustering was 
noted in the unweighted UniFrac analyses (supplementary table 2) and the colon microbiota was characterized by reduced diversity (Observed and PD) from cyanocobalamin supplementation ( $\mathrm{n}=7-12 ; P$ $<0.05)$.

a) naïve_CON vs. naive_CNCbl40

\begin{tabular}{|c|c|c|}
\hline Ileum & $\begin{array}{l}\text { [ASV32] O scillospiraceae } \\
\text { [ASV131] Lach nospiraceae } \\
\text { [ASV87] Lachnospira ceae } \\
\text { [ASV11] Erysipelatodo stridiaceae } \\
\text { [ASV158] Erssipelotrichace ae } \\
\text { [ASV184 Entero habdus } \\
\text { [ASV5] Muribaculacease }\end{array}$ & $\begin{array}{l}\text { [ASV8] Lachnospira ceae NK4A136 group } \\
\text { [ASV11] Lachnospiraceae NK4A136 group } \\
\text { [ASV131] Lachnospiraceae } \\
\text { [ASV7] Citrobacter rodentium } \\
\text { [ASV319] Lachnospiraceae }\end{array}$ \\
\hline Cecum & 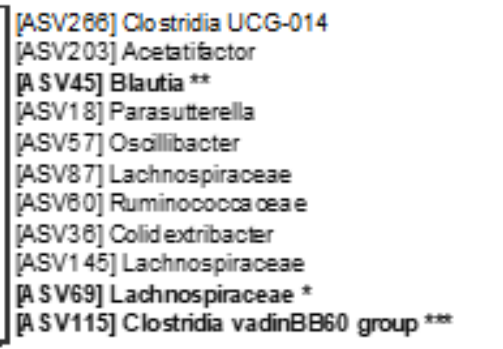 & 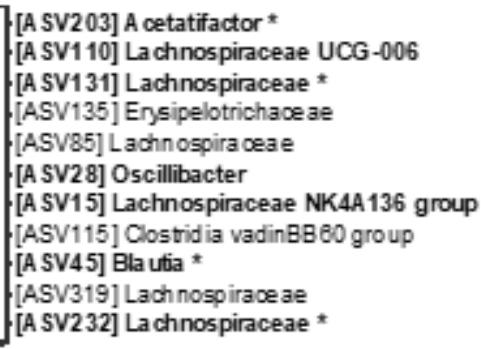 \\
\hline Colon & 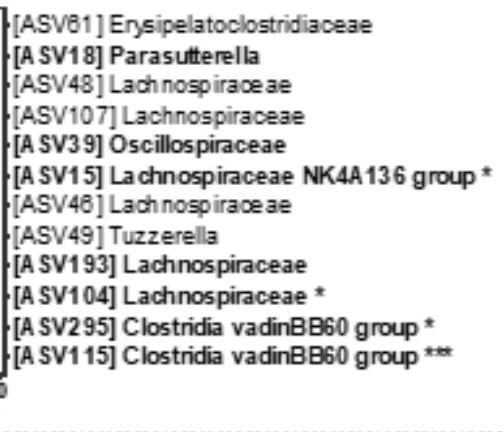 & 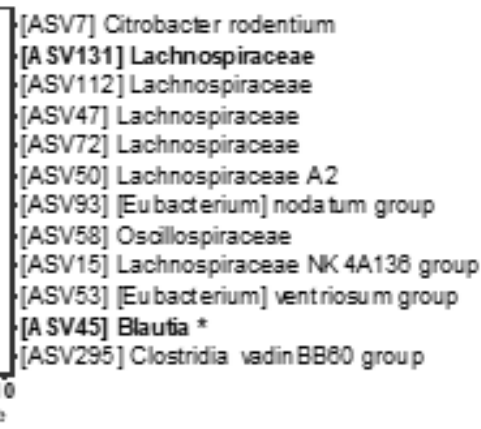 \\
\hline
\end{tabular}

\section{Figure 3}

Cyanocobalamin supplementation altered the Firmicutes population throughout the gastrointestinal tract in $\mathrm{C} 3 \mathrm{H} / \mathrm{HeOuJ}$ mice as determined by DESeq2 differential expression. The control group had a greater abundance of Firmicutes, including Lachnospiraceae species and Clostridia vadinBB60 group bacterium in the cecum and colon (a) pre-infection and (b) post-infection ( $n=6-8$; only ASVs with a $P$-value less than 0.05 were plotted; adjusted $P$-value were used for significance; bolded taxa represent a trend $(P<$ $0.10) ; * P<0.05$, ** $P<0.01$, *** $P<0.001)$. 


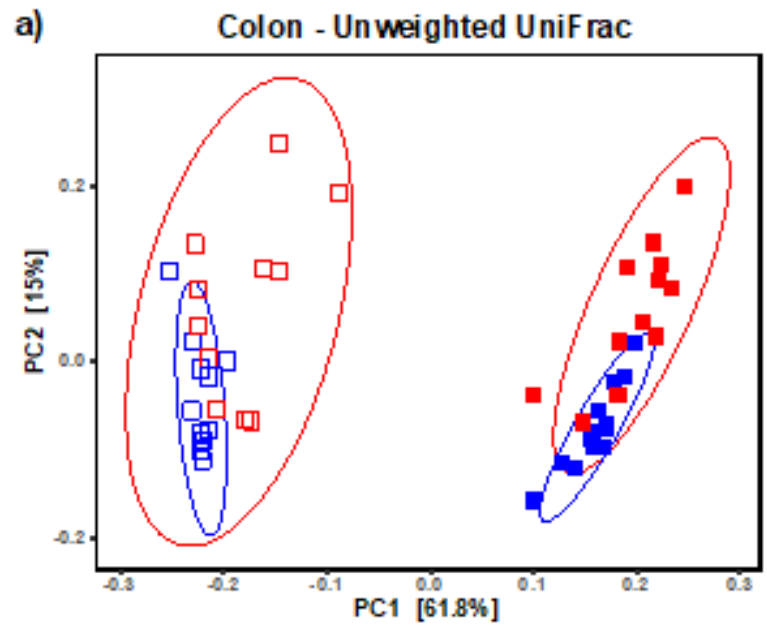

b)

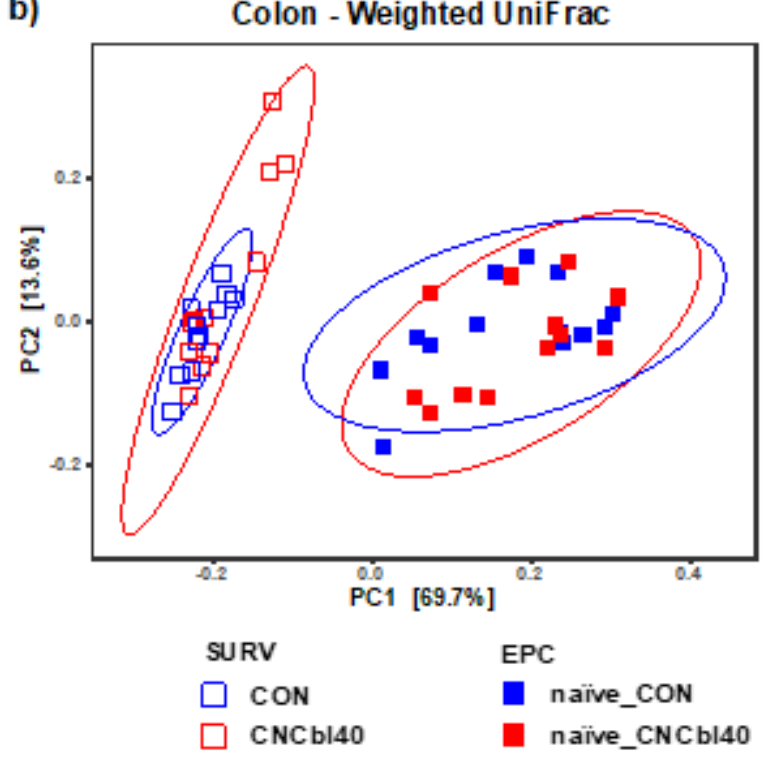

c)

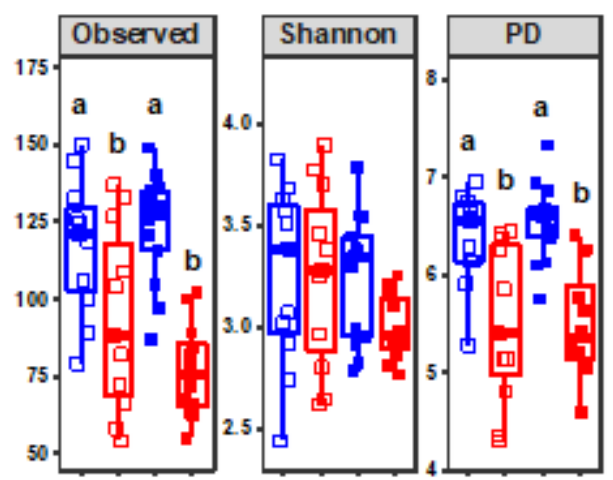

d) Colon - CON vs. CNC bl40 [SU RV]

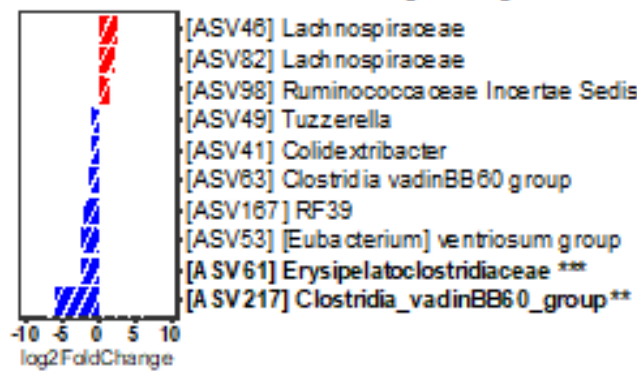

e) Colon - naïve_CON vs. naiive_C NCbI40 [E PC]

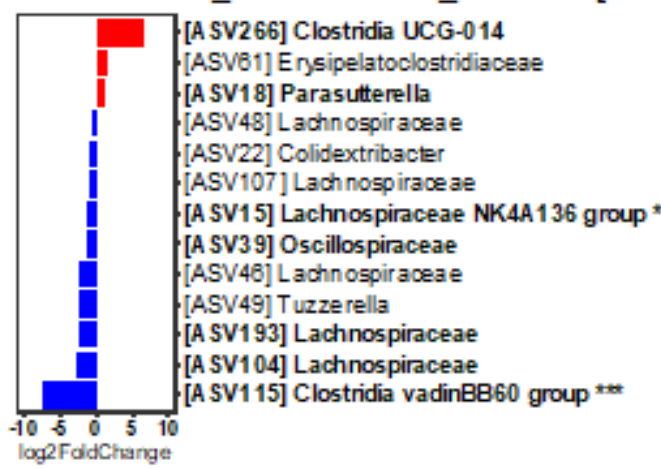

Figure 4

Comparison of microbial communities in naïve $\mathrm{C} 3 \mathrm{H} / \mathrm{HeO} \mathrm{J}$ mice from SURV and ECP experiments. (a) Unweighted UniFrac PCoA plot and (b) weighed UniFrac comparison shows a similar pattern in microbial community clustering (see supplementary table 2). (c) The changes were associated with a consistent reduction in alpha diversity (Observed and $P D)$ regardless of experiment $(\mathrm{n}=10-12 ; P<0.05)$. Differential expression determine by DEseq2 analysis also showed that the Firmicute populations (Clostridia vadinBB60 group) were impacted by cyanocobalamin in both mice harboring (d) SURV and (e) EPC gut microbiomes ( $\mathrm{n}=10-12$; bolded taxa represent a trend $(P<0.10 ; * P<0.05$, ** $P<0.01$, *** $P<0.001)$. 
a)

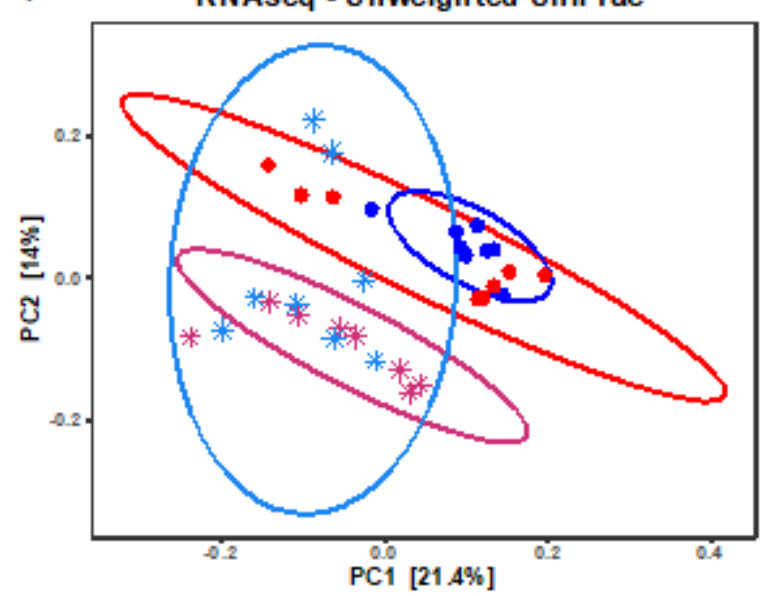

b)

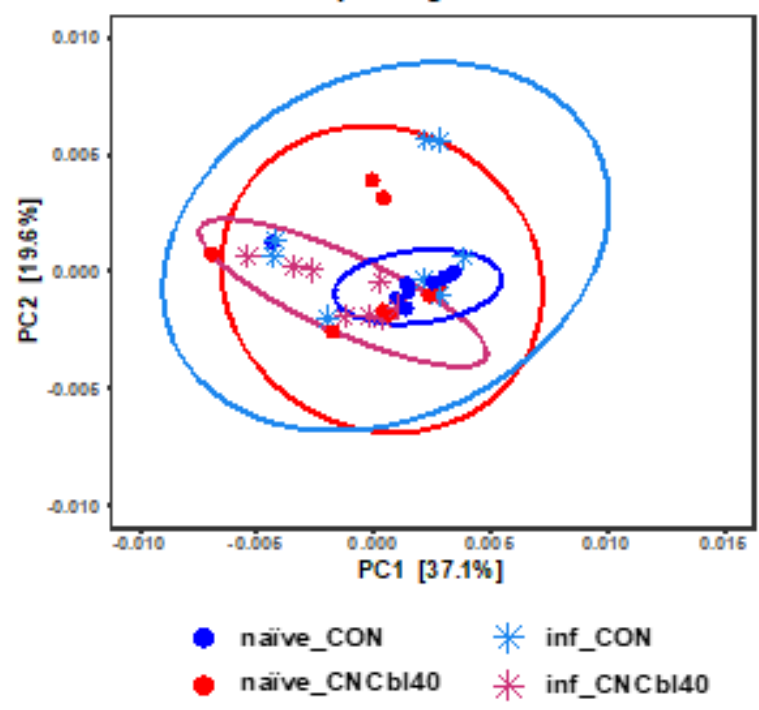

c)

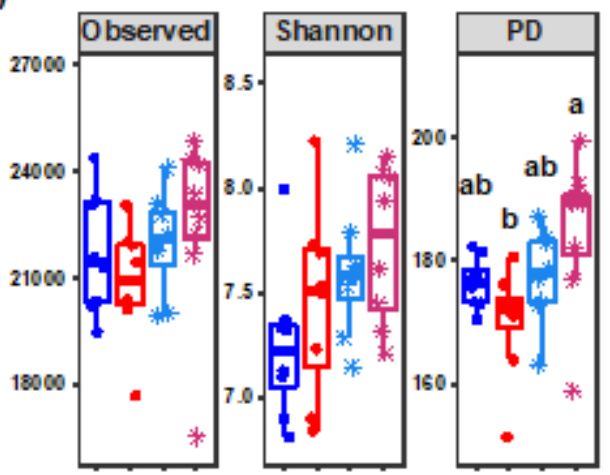

d)

naive_CON vs. naïve_CNCb140

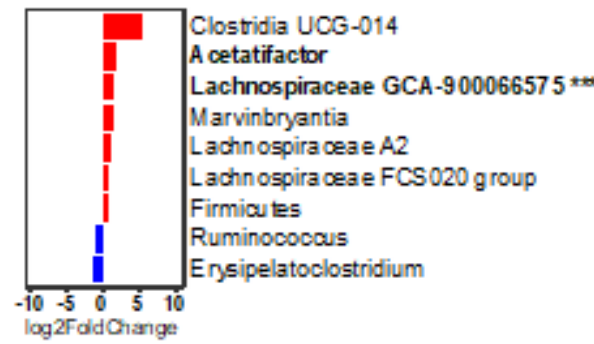

e) inf_CON vs. inf_CNCbI40

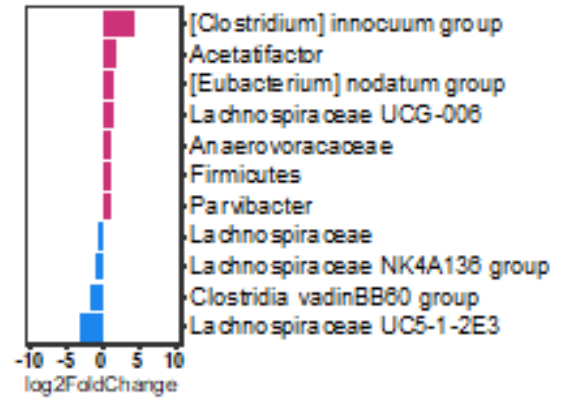

Figure 5

Cecal microbiota analysis of ribosomal rRNA data pulled from SAMSA2 metatranscriptome analysis. Distinct clustering of microbial communities in (a) unweighted but not (b) weighted UniFrac PCoA plots (see supplementary table 2). (c) Alpha diversity as determine by Observed, Shannon and PD metrics showed that diversity (PD only) increased post-infection for cyanocobalamin-supplemented mice compared to control $(P<0.05)$. Differential expression analysis using DEseq2 of microbial taxa confirmed that the Firmicutes populations were altered from cyanocobalamin supplementation in (d) naïve and $(e)$ infected mice $(n=6-8$; bolded taxa represent a trend $(P<0.10)$; $* \star \star P<0.001)$. 
a) naive_CON vs. naive_CNCb 140

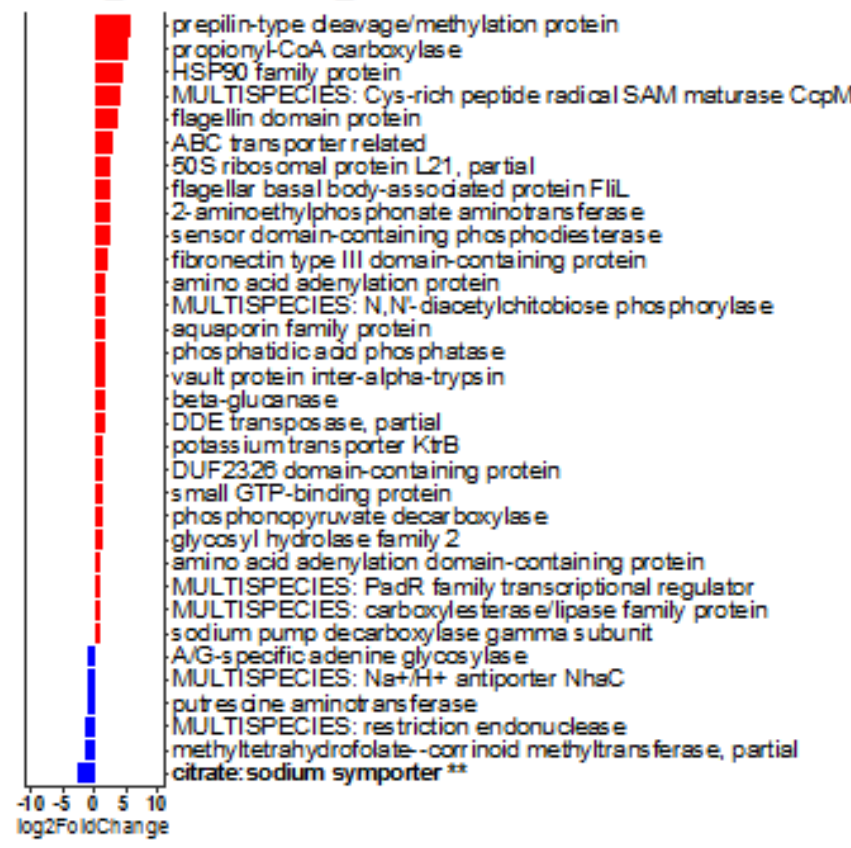

b) inf_CON vs. inf_CNCbl40

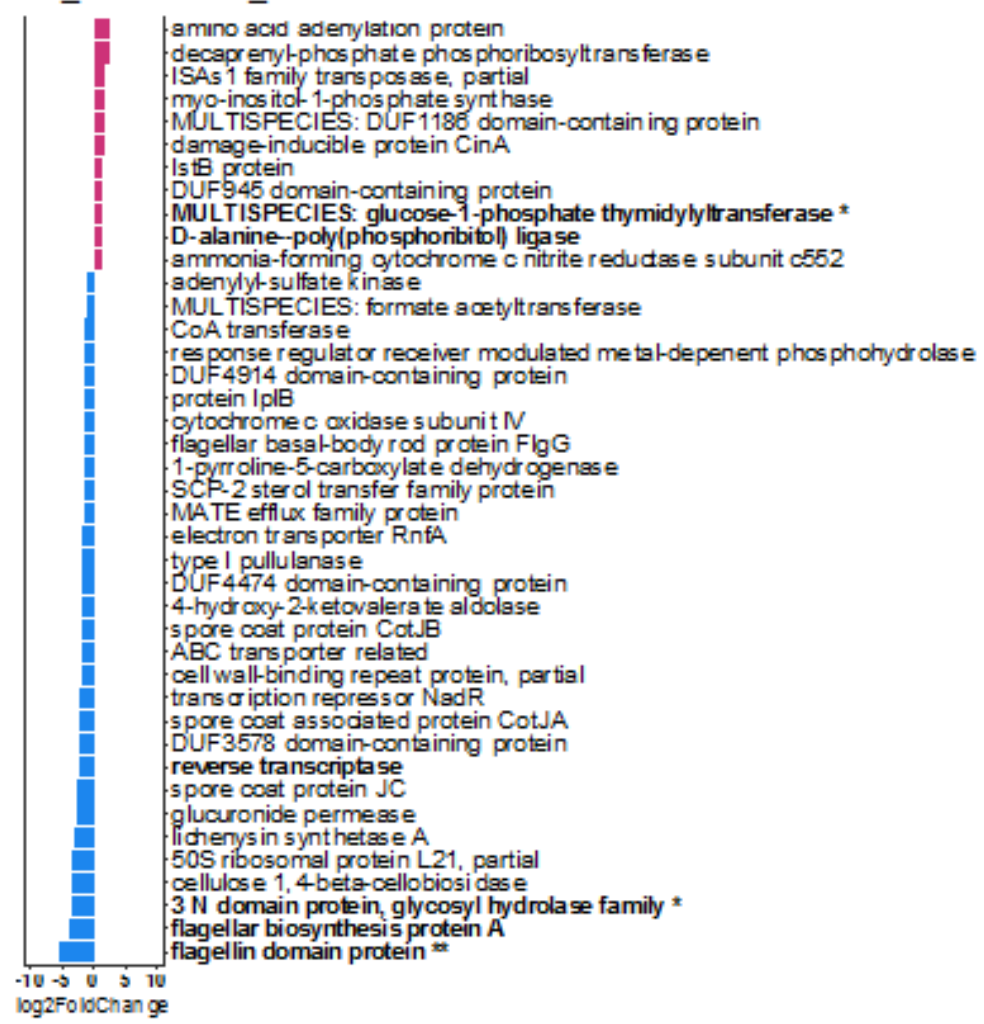

\section{Figure 6}

Cecal metatranscriptome analysis revealed key changes in the overall activity of the microbiota. (a) Naïve and (b) C. rodentium-challenged mice supplemented with cyanocobalamin displayed altered functional activities related to metabolism (Citrate:sodium symporter) and motility (Flagellin domain protein), which was related to the Firmicute populations $(\mathrm{n}=8$; bolded taxa represent a trend $(P<0.10)$; * $P<0.05$, ** $P<$ $0.01)$.

a)

Subsy stems L3 naïve_CON vs. naïve_CNCb140

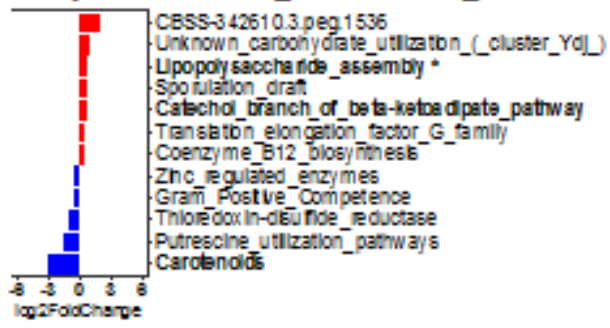

1.

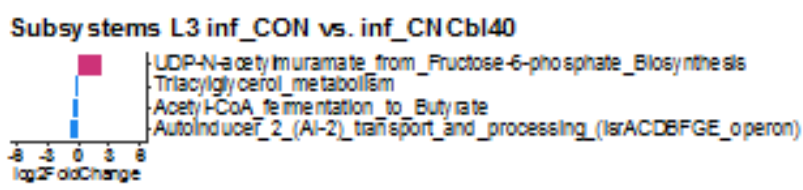

Figure 7 
Pathway analysis of the $\mathrm{C} 3 \mathrm{H} / \mathrm{HeOuJ}$ cecal microbiota's functional activity using the SEED subsystems at level 3 from SAMSA2 pipeline. Cyanocobalamin supplementation altered various increased pathways related to Gram-negative while decreasing genes related to Gram-positive bacteria in (a) naïve mice but not in (b) $C$. rodentium-challenged mice $(\mathrm{n}=8$; bolded taxa represent a trend $(P<0.10)$; * $P<0.05)$.

a)

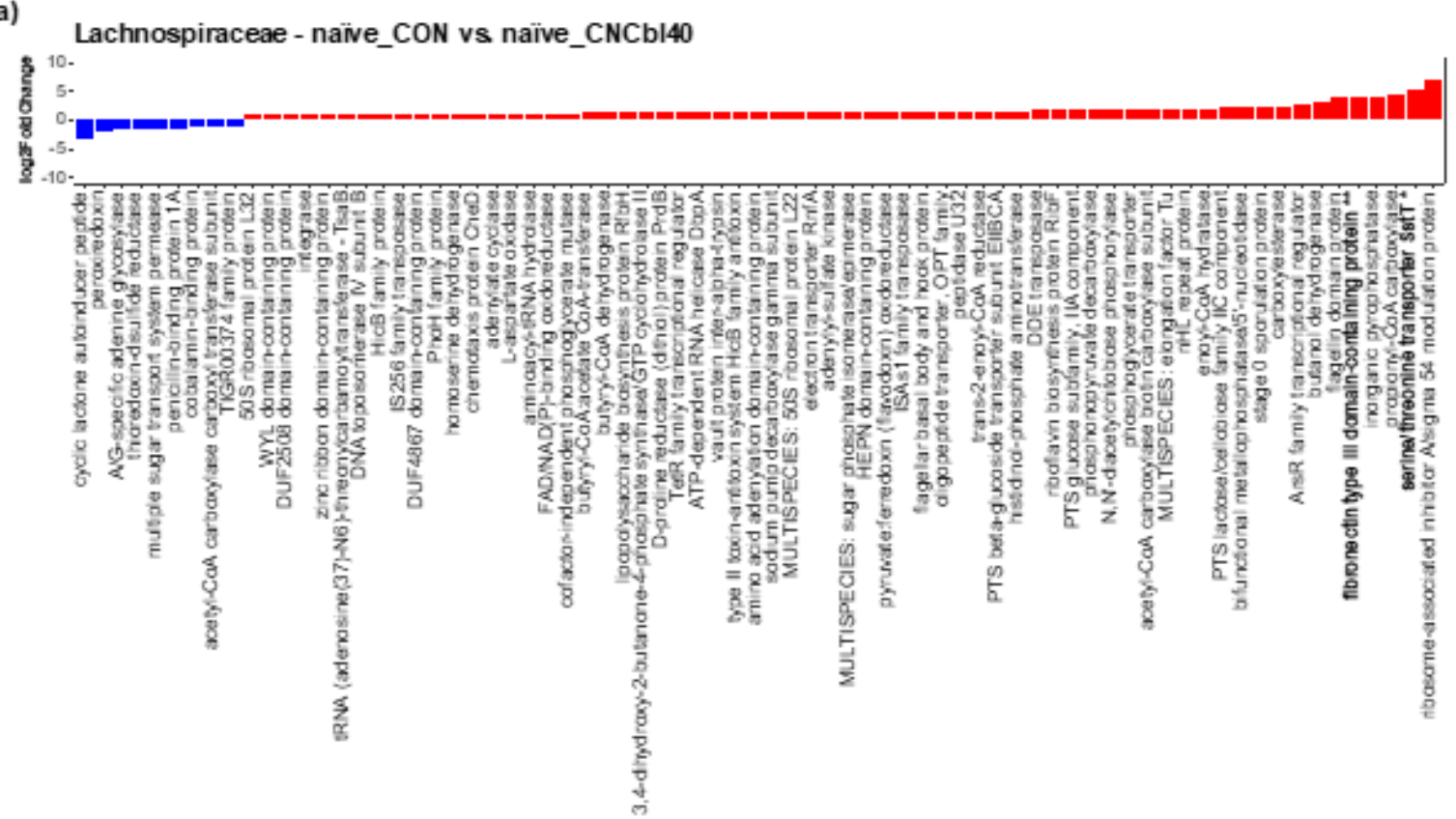

b)

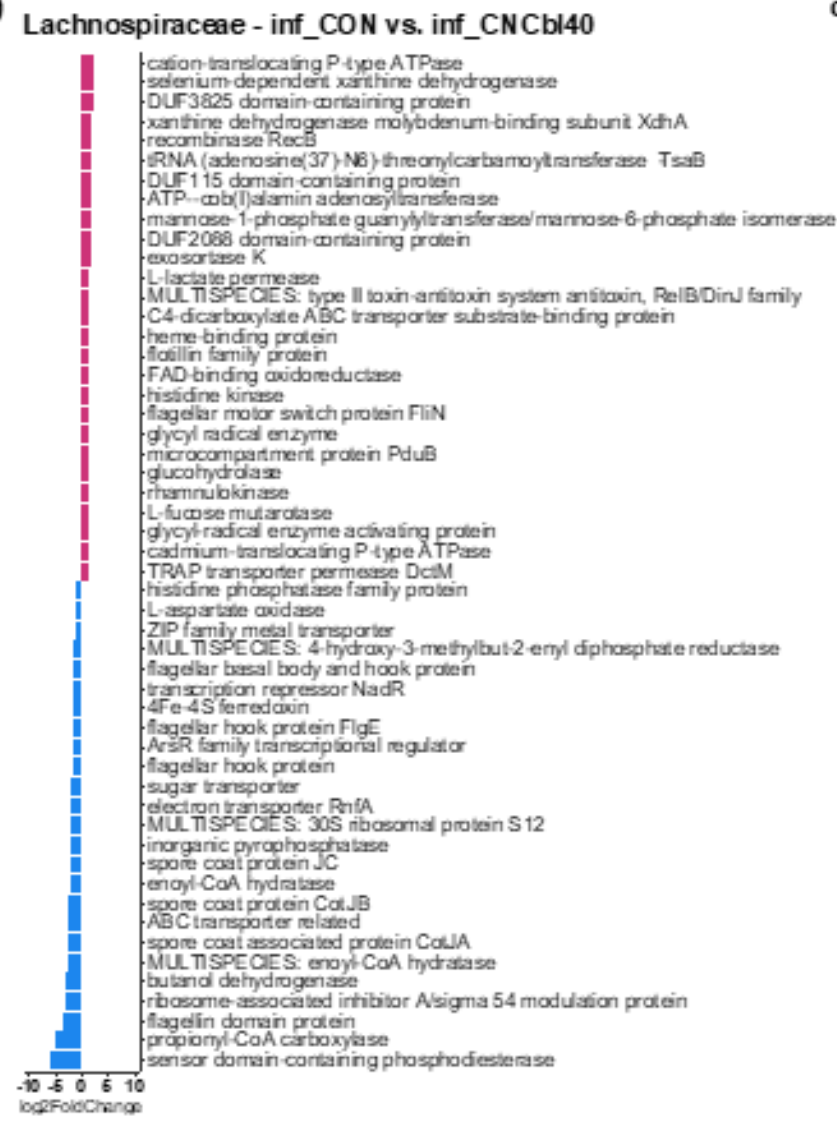

c)

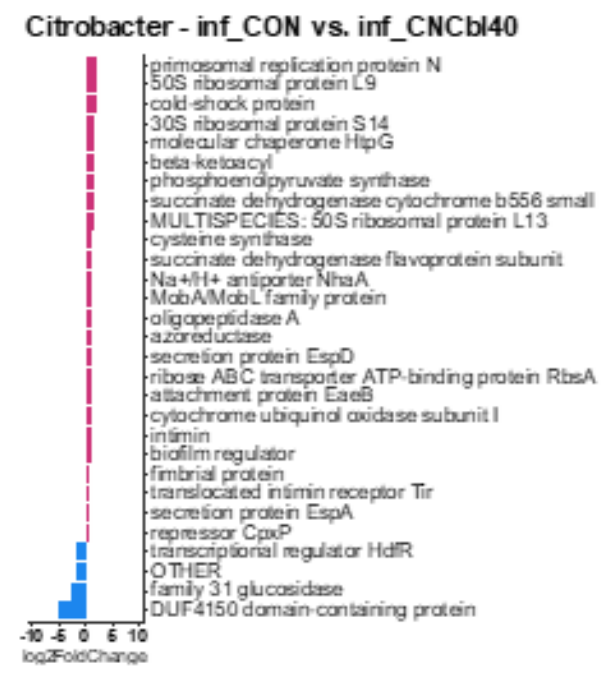

\section{Figure 8}


Filtered analysis of cecal metatranscriptomic data revealed that cyanocobalamin supplementation impacted the functional activity of Lachnospiraceae family members and Citrobacter rodentium. Prior to infection, the functional activity of the Lachnospiraceae family (a) shows that cyanocobalamin treatment increased the expression of numerous genes, including fibronectin type III domain-containing protein and serine/threonine transport SstT. (b) After exposure to $C$. rodentium, the Lachnospiraceae family members of the inf_CON and inf_CNCbl40 groups displayed distinct activities. Citrobacter specific gene expression (c) was more pronounced in the inf_CNCbl 40 group than inf_CON with notable signals of increased virulence gene expression, while control mice had more family 31 glucosidase activity $(n=8$; bolded taxa represent a trend $(P<0.10)$; * $P<0.05$, ** $P<0.01)$.

a)

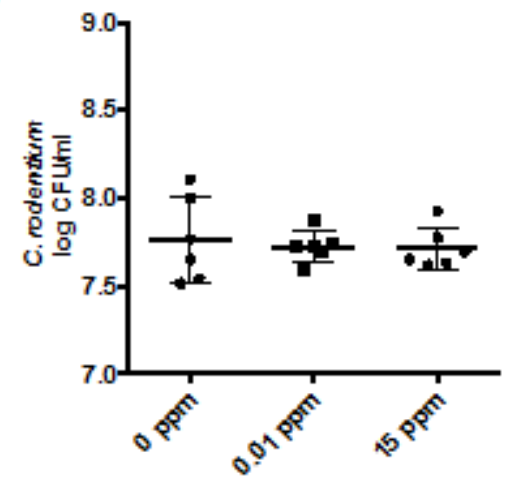

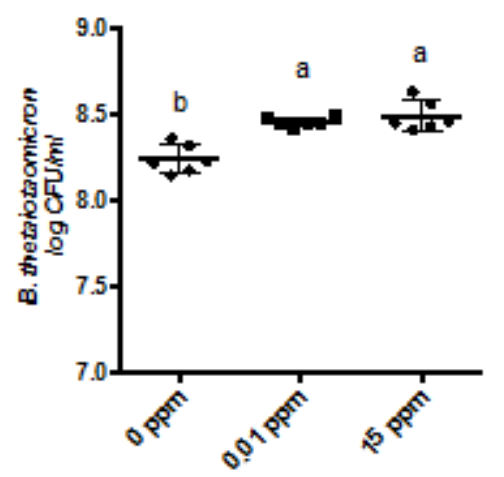

c)

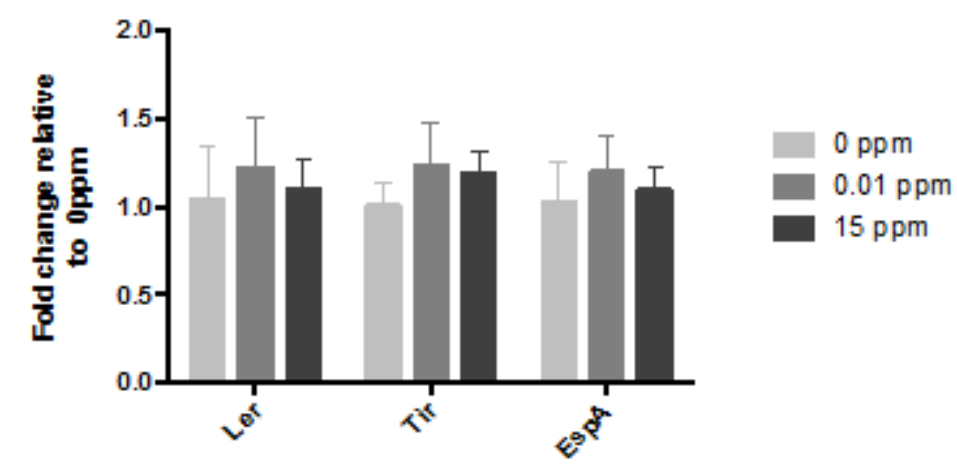

b)

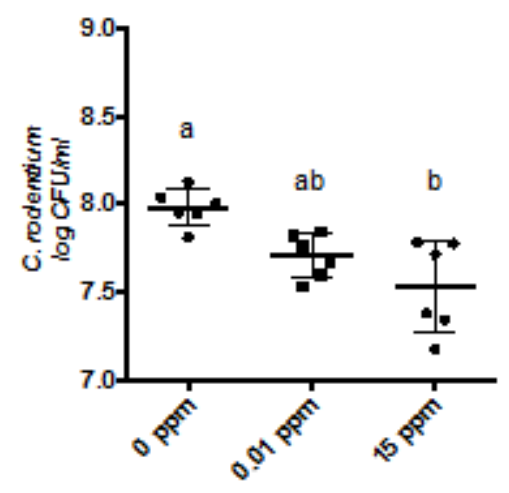

d) C. rodentium alone

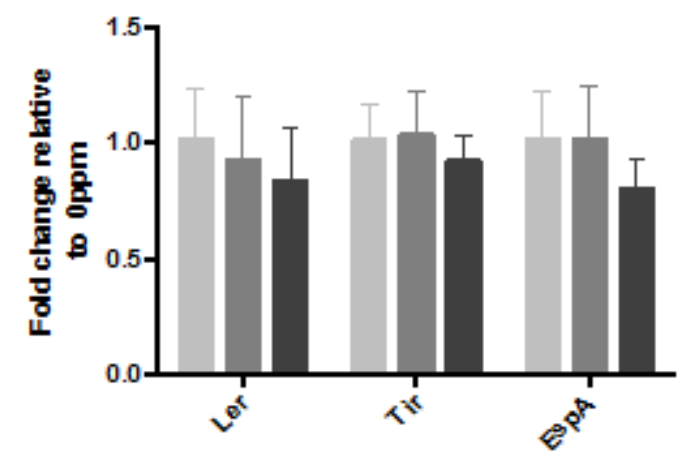

Figure 9

Cyanocobalamin alone was unable to directly alter $C$. rodentium growth or virulence in vitro at physiological relevant concentrations. (a) Enumeration of $C$. rodentium and $B$. thetaiotaomicron grown in competition for $6 \mathrm{~h}$ anaerobically, and (b) of $C$. rodentium grown alone. Expression of gene related to virulence (Ler, Tir, and EspA) did not differ between treatments when $C$. rodentium was grown in (c) competition or (d) alone at different concentration of cyanocobalamin $(\mathrm{n}=6 ; P<0.05)$. 
a)
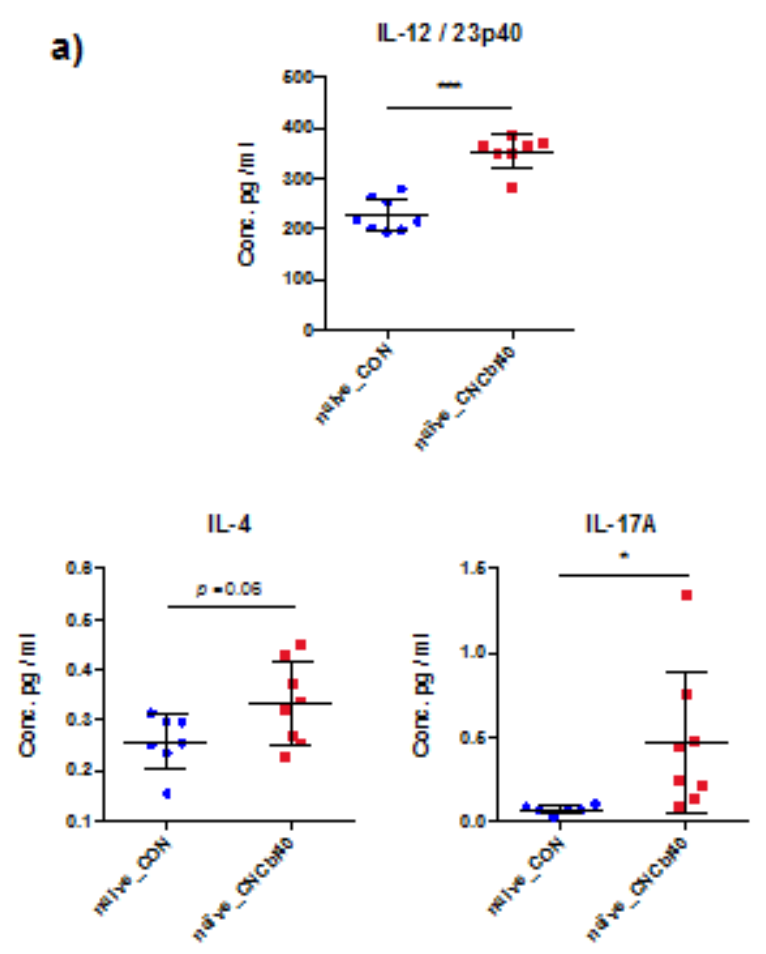

b)
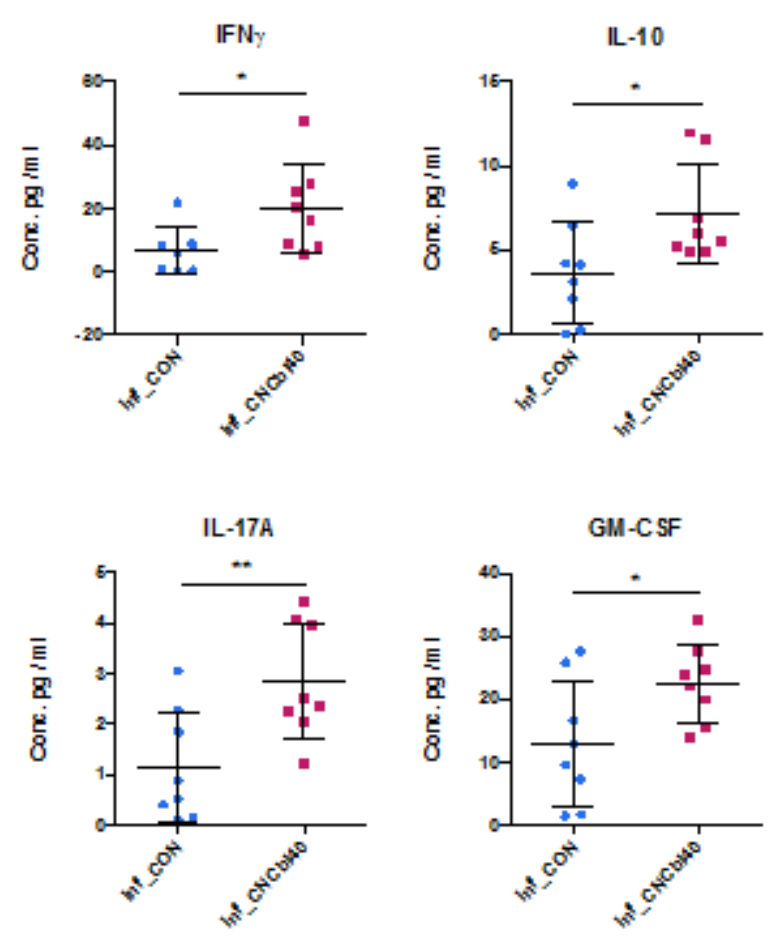

\section{Figure 10}

Significant host biomarker concentrations determined in colon tissue homogenates of $\mathrm{C} 3 \mathrm{H} / \mathrm{HeOuJ}$ mice. (a) Cyanocobalamin supplementation enhanced IL-12/23p40, and IL-17A cytokines of naïve mice, with a higher trend of IL-4 levels $(P=0.06)$ compared to control. (b) $C$. rodentium challenge led to a significant increase in IFNg, IL-10, IL-17A, and GM-CSF in the cyanocobalamin-supplemented mice $(\mathrm{n}=8 ; * P<0.05$, $\star \star P<0.01, * \star \star P<0.001)$. 
a)

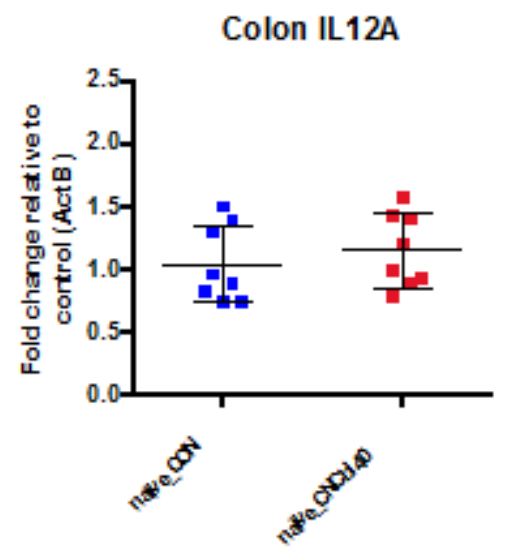

c)

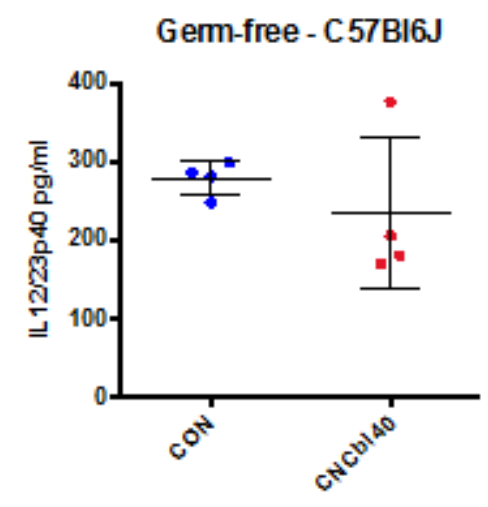

Colon IL 12B

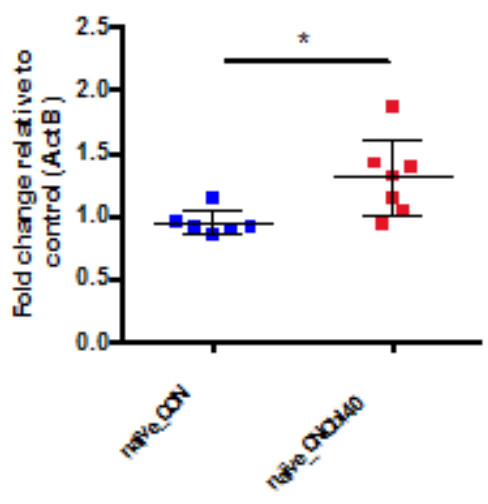

b)

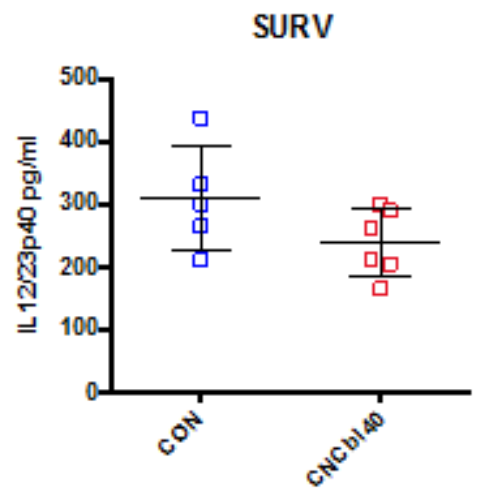

\section{Figure 11}

d)

Conventional - C57BI6J

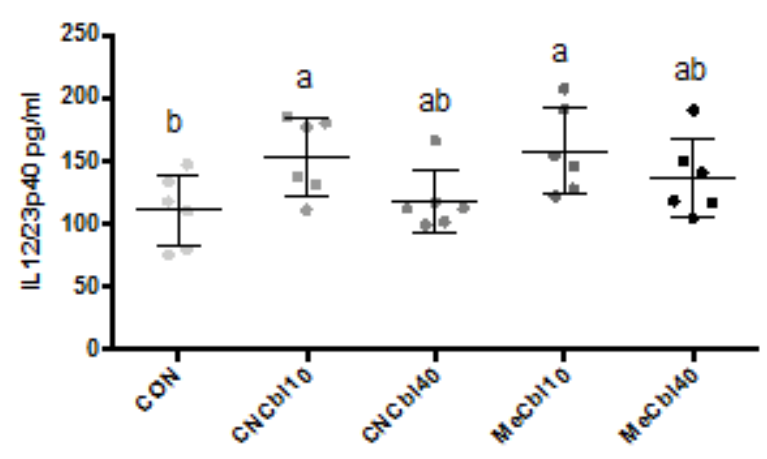

Pre-infection levels of IL-12/23p40 in colon tissues were directly related to microbiota structure and independent of related mice genotypes. (a) Gene expression was significantly higher for IL12B, the p40 subunit coding gene, but not for the IL12A (p35) gene $(n=8)$ in the EPC experiment. (b) The SURV experiment did not display the increase in IL12/23p40 ( $n=5-6)$, nor did (c) germ-free C57BI/6J mice $(n=$

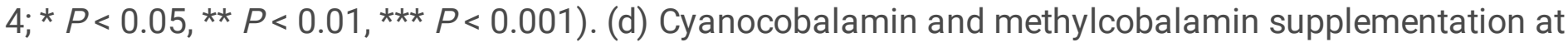
$10 \mu \mathrm{g} / \mathrm{ml}$ and $40 \mu \mathrm{g} / \mathrm{ml}$ increased IL/23p40 protein levels in the colon of conventional C57BI/6J mice ( $\mathrm{n}$ $=6 ; P<0.05)$ compared to control.

\section{Supplementary Files}

This is a list of supplementary files associated with this preprint. Click to download.

- Supplementaryinformation.docx 